\title{
LE AUTORITÀ INDIPENDENTI IN ITALIA COME TEMA DI RICERCA
}

\author{
di Massimo Morisi
}

\section{Introduzione}

Le funzioni di cui si occupano le autorità indipendenti nel nostro paese, formano un elenco abbastanza impressionante. A tali istituzioni viene infatti affidata una complessa gamma di funzioni decisionali in una molteplicità di settori di regolazione economica e sociale di cruciale importanza. Moneta e credito $^{1}$, concorrenza ${ }^{2}$ e assicurazioni ${ }^{3}$, borsa, valori mobiliari ${ }^{4}$ e fondi pensione ${ }^{5}$, comunicazioni di massa ${ }^{6}$ e informatica pub-

1 Dalle norme poste dal R.D. 11 giugno 1936, n. 1067, Approvazione dello statuto della Banca d'Italia; dalla legge 7 febbraio 1992, n. 82, Modificazioni alle procedure stabilite dal T.U. sugli istituti di emissione e sulla circolazione dei biglietti di banca, approvato con R.D. n. 204/1910, in materia di variazioni del tasso ufficiale di sconto e dell'interesse sulle anticipazioni; dal d.lgs. $1^{\circ}$ settembre 1993, n. 385, Testo Unico delle leggi in materia bancaria e creditizia.

2 Per le norme della legge 10 ottobre 1990, n. 287, Norme per la tutela della concorrenza e del mercato, e del d.lgs. 25 gennaio 1992, n. 74, Attuazione della direttiva (Cee) $n .450 / 84$, in materia di pubblicità ingannevole.

3 Vedi le leggi 12 agosto 1982, n. 576, Riforma della vigilanza sulle assicurazioni; 9 gennaio 1991, n. 20, Integrazioni e modifiche alla legge 12 agosto 1982, n. 576, e norme sul controllo delle partecipazioni di imprese o enti assicurativi; e il d.p.r. 18 aprile 1994, n. 385, Regolamento recante semplificazione dei procedimenti amministrativi in materia di assicurazioni private e di interesse collettivo di competenza del Ministero dell'Industria, del Commercio e dell'Artigianato.

4 Vedi il d.l. 18 aprile 1974 , n. 95 , convertito con modificazioni nella legge 7 giugno 1974, n. 216, Disposizioni relative al mercato mobiliare ed al trattamento fiscale dei titoli; la legge 17 maggio 1991, n. 157, Norme relative all'uso di informazioni riservate nelle operazioni in valori mobiliari e alla Commissione nazionale per la società e la borsa; la legge 2 gennaio 1991, n. 1, Disciplina dell'attività di intermediazione mobiliare e disposizioni sull'organizzazione dei mercati mobiliari.

5 Vedi il d.lgs. 21 aprile 1993, n. 124, Disciplina delle forme pensionistiche complementari, a norma dell'articolo 2, comma 1, lettera v) della legge 23 ottobre 1992, $n$. 421, successivamente modificato e integrato dal d.lgs. 30 dicembre 1993, n. 585, e dalla legge 8 agosto 1995, n. 335, di riforma del regime pensionistico.

6 In base alle leggi 5 agosto 1981, n. 416, Disciplina delle imprese editrici e provvidenze per l'editoria; 6 agosto 1990, n. 223, Disciplina del sistema radiotelevisivo pubblico

RIVISTA ITALIANA DI SCIENZA POLITICA / a. XXVII, n. 2, agosto 1997 
blica $^{7}$, appalti di lavori pubblici ${ }^{8}$ e tariffe di energia elettrica e gas $^{9}$, scioperi nei servizi pubblici ${ }^{10}$ e contrattazione sindacale nelle pubbliche amministrazioni ${ }^{11}$, monitoraggio e innovazione nell'ambiente $^{12}$ e nel funzionamento del sistema sanitario ${ }^{13}$ sono solo i più evidenti items di un profondo spostamento di poteri dall'apparato politico e amministrativo di governo verso una rete articolata, e in continua diramazione, di nuove e separate arene di rappresentazione, intermediazione o aggiudicazione, secondo i casi, di interessi. Chi domani volesse impegnarsi in ricerche empiriche sulle politiche pubbliche in questi settori decisivi per il sistema economico e sociale di questo paese, dovrebbe primariamente sottoporre ad analisi l'azione delle autborities e le reti di relazioni con gli interessi organizzati e diffusi in cui esse sono chiamate ad operare. Il campo visivo della rilevazione andrebbe profondamente riorientato. Solo ex post andrebbe ponderato il ruolo delle istituzioni classiche della forma di governo, come i partiti, gli organi dell'Esecutivo e il Parlamento. L'analisi empirica, a fronte di casi e circostanze determinate, potrebbe anche sancirne la (de) qualificazione alla sola stregua di attori e arene integrative.

Tuttavia, osservata l'importanza crescente e virtualmente pervasiva del fenomeno ${ }^{14}$ e del suo ruolo nella trasformazione

e privato; 10 dicembre 1993, n. 515, Disciplina delle campagne elettorali per l'elezione alla Camera dei deputati ed al Senato della Repubblica.

7 Vedi il d.lgs. 12 febbraio 1993, n. 39, Norme in materia di sistemi informativi automatizzati.

8 Vedi la legge 11 febbraio 1994, n. 109, Legge quadro in materia di lavori pubbli$c i$, modificata dal d.l. 18 dicembre 1995, n. 535 .

9 In base alla legge 14 novembre 1995, n. 481, Norme per la concorrenza e la regolazione dei servizi di pubblica utilità. Istituzione delle Autorità di regolamentazione dei servizi di pubblica utilità.

10 In base alla legge 12 giugno 1990, n. 146, Norme sull'esercizio del diritto di sciopero nei servizi pubblici essenziali e sulla salvaguardia dei diritti della persona costituzionalmente tutelati. Istituzione della Commissione di garanzia dell'attuazione della legge.

11 Secondo il d.lgs. 3 febbraio 1993, n. 29, Razionalizzazione dell'organizzazione delle amministrazioni pubbliche; e il d.p.r. 25 gennaio 1994, n. 144 (modificato dal d.p.r. 21 settembre 1994, n. 610), Regolamento recante norme per l'organizzazione ed il funzionamento dell'Agenzia per la rappresentanza negoziale delle pubbliche amministrazioni.

12 In base al d.l. 4 dicembre 1993, n. 496, convertito con modificazioni nella legge 21 gennaio 1994, n. 61, Disposizioni urgenti sulla riorganizzazione dei controlli ambientali ed istituzione della Agenzia nazionale per la protezione dell'ambiente.

13 In base al d.lgs. 30 giugno 1993, n. 266, Riordinamento del Ministero della Sanità a norma dell'art. 1, comma 1, lettera b) della legge 23 ottobre 1992, n. 421, art. 5; e al d.m. 22 febbraio 1994, n. 233, Regolamento per la organizzazione, il funzionamento e la disciplina delle attività dell'Agenzia per $i$ servizi sanitari regionali.

14 Che non è certo esente, a sua volta, da fenomeni e propensioni di «moda», di 
dello Stato e dei connessi mutamenti nelle interazioni tra apparati statuali e politiche pubbliche (Majone e La Spina 1990, 1991 e 1992a; La Spina 1996; Ferrari 1997), occorre anche constatare come non esista un'unica radice storica cui ancorare comparativamente un simile processo innovativo. Ad esempio, quando volgiamo il nostro sguardo al di là dell'Atlantico, secondo una comparazione rituale su questo tema, vediamo subito che, nell'Europa di oggi, il diffondersi di autorità indipendenti si deve in notevole misura ai processi di privatizzazione e alla correlativa propensione dello Stato a ritrarsi dal mercato, dismettendo un ruolo di diretto operatore industriale e finanziario, per accentuare, invece, la propria funzionalità regolatoria, secondo quello che è ormai un postulato ideologico, ipostatizzato prescrittivamente nel processo di integrazione europea e nelle sue scansioni normative e giurisprudenziali. Invece, nella tradizione storica degli Stati Uniti, che il senso comune identifica con la madre della nozione stessa di authority e di agency, tutto risale al corposo tentativo di estendere, verso la fine dello scorso secolo, l'intervento del potere pubblico a sostegno e a tutela del mercato in settori cruciali come gli scambi commerciali, la borsa, le telecomunicazioni. Per converso, sappiamo che se proprio l'onda lunga della ritrazione dello Stato europeo dal concorso imprenditoriale pubblico alla espansione e alla conformazione del mercato e alle sue dinamiche ha promosso e motivato il proliferare delle autorità indipendenti, nel caso americano l'omologo processo storico di ridimensionamento del potere pubblico ha prodotto la crisi e la critica di quel medesimo ge-

cui il dibattito quotidiano sui media circa le crisi dell'attuale congiuntura economica e politico-istituzionale italiana, fornisce esempi infiniti, quasi che nessuna ricetta innovativa, nei campi più svariati, possa esimersene per essere credibile, e quasi che «autorità indipendente» sia garanzia dell'attendibilità della proposta e della politica specifica che si propone. E se, per caso, di ricorso a un qualche «intervento pubblico» si parla, sembra oggi «politicamente corretto» farlo solo nella chiave, appunto, di una qualche correlativa autorità indipendente. Un esempio tra i moltissimi: «Non si può dire ai troppi giovani che cercano invano lavoro di pazientare ancora. Alla fine si spareranno o spareranno a noi»: su questa premessa Cesare Romiti propone la «istituzione di un'Alta autorità per gli investimenti in infrastrutture, un organismo costituito dal governo che accorpi le competenze dei singoli ministeri e superi le competenze degli enti locali e che, a partire dalle province con una disoccupazione oltre il $10 \%$, faccia una verifica sulle grandi opere, ne gestisca la realizzazione, ne progetti di nuove. Eliminando vincoli burocratici e amministrativi, canalizzando i finanziamenti statali, comunitari e privati. Sarebbe poi possibile concordare a livello europeo che questi "costi" di investimento per lo Stato, come è per le aziende, siano esclusi dal conto economico, cioè dal bilancio pubblico. In sostanza, si potrebbe utilizzare da subito quell'elasticità "politica" che il Trattato prevede» (Romiti 1996). 
nere di istituzioni ${ }^{15}$. Inoltre, a differenza delle più tarde configurazioni europee, negli Stati Uniti troviamo una diretta correlazione tra la configurazione istituzionale e organizzativa delle autorità indipendenti e la forma di governo, per cui le funzioni regolative di quelle istituzioni sono concepite come un prolungamento funzionale, specifico e continuativo delle competenze legislative del Congresso ${ }^{16}$. Resta vero, tuttavia, che lo stampo istituzionale nordamericano è l'ispiratore di una serie di profili tendenzialmente comuni e di particolare longevità. Ad esempio, la composizione collegiale dei diversi istituti ne è un diretto portato, finalizzato a conferire a tali istituzioni un segno almeno virtuale di autonomia; così, ancora, si può dire per il confluire su tali istituti di poteri sia di regolazione che di aggiudicazione circa i casi concreti da dirimere; e così, soprattutto, per l'assenza di un potere preventivo di indirizzo, o successivo di controllo, nelle mani dell'Esecutivo ${ }^{17}$.

In Europa gli esempi più emblematici li rinveniamo nell'esperienza inglese ed in quella francese. Le Quasi Autonomous non Governamental Organizations, designate con l'acronimo «Quangos», colpiscono l'immaginario sulla prima delle due esperienze nazionali. In realtà, si tratta di un «polimorfo calderone» (Amato 1996, 4) costituito da centinaia di entità, moltiplicatesi con il governo della signora Thatcher al fine tendenziale di controbilanciare e ridimensionare il potere degli enti locali, ove vengono assemblati $i$ consigli e $i$ comitati di natura sia tecnica, sia democratico-categoriale, sia scientifica, sia culturale che già affiancavano le pubbliche amministrazioni, e a cui è stata riconosciuta, in virtù della loro conformazione tecnico-rap-

15 Cfr., emblematicamente, il caso del Civic Aereonautic Board, il cui smantellamento viene in proposito ricordato da Amato (1996).

16 «In quella forma di governo, infatti, quando la legge prefiguri una missione in primo luogo di regolazione (continuativa) di un settore, tale missione appare piuttosto come una estensione di quella legislativa del Congresso, che non un compito dell'Esecutivo. Di qui la sua attribuzione non a questo, ma a dirette emanazioni dell'organo legislativo» (Amato 1996, 3).

17 Sono elementi conformativi che delineano un profilo costitutivo originario già nella Interstate Commerce Commission istituita nel 1887 (la cui indipendenza viene conseguita pienamente poco più tardi, nel 1889) per regolare tariffe e comportamenti delle compagnie ferroviarie a tutela soprattutto dei produttori agricoli e dei commercianti nel trasporto dei loro prodotti; e lo saranno anche per la Federal Trade Commission, nata per regolare e colpire i comportamenti anticoncorrenziali; per la Federal Communication Commission, chiamata a regolare l'uso dell'etere; per la Securities and Exchange Commission, destinata a vigilare sulla borsa; e per le altre che seguiranno - per le quali cfr. Ferrari (1997). 
presentativa, una parziale autonomia dalla capacità di direzione e di controllo dei ministri sugli apparati ministeriali (Machin 1989; Wilson 1995). Tuttavia, nello stesso novero di Quangos, troviamo autorità propriamente indipendenti come la Indipendent Broadcasting Authority, titolare di funzioni analoghe a quelle del nostro Garante per l'editoria e la televisione e del Conséil francese per l'Audiovisuel, e che cumula su di sé l'esigenza di regolare attività economiche capaci di condizionare la libertà di pensiero, mediante - per ciò stesso - una istituzione che mira a sottrarne la garanzia e l'esercizio alla discrezionalità del potere politico. $\mathrm{Ma}$, a parte i Quangos, il caso della Gran Bretagna - oltre alle Next Steps Agencies ${ }^{18}$ - annovera la gamma delle autorità indipendenti create nel corso dell'ultimo decennio nell'ambito dei processi di privatizzazione dei pubblici servizi (elettricità, gas, telecomunicazioni) già esercitati - per lo più in regime di monopolio - da imprese pubbliche sottoposte all'indirizzo politico del ministero. La loro ratio è evidente e comune a quella delle omologhe esperienze europee: accompagnare e supportare la transizione dalla titolarità pubblica a quella privatistica delle imprese con una regolazione pubblica ma non governativa né politico-elettorale, fondata piuttosto su una expertise tecnica che scolleghi le strategie di impresa e di mercato dai cicli politici di governo, così da sostituire i monopoli con mercati competitivi di settore ${ }^{19}$. La loro indipendenza risente comunque del loro innesto sulla forma di governo britannica, per cui i poteri di nomina permangono pur sempre in capo all'Esecutivo e le funzioni di vigilanza sono ancorate al Parlamento il quale, con le proprie commissioni competenti, convoca direttamente i responsabili delle varie Authorities, «interroga e fa comunque emergere giudizi e orientamenti, pur al di fuori di responsabilità formali» (Amato 1996, 5).

Nel modello francese, le autorità indipendenti sono principalmente funzionalizzate alla «tutela dei diritti dei cittadini da realizzare in forme più continuative di quelle giudiziarie, ma $\mathrm{da}$ sottrarre - proprio in quanto diritti - alle variabili degli indiriz-

18 Per le quali cfr. Perez (1991; 1996b).

19 «Si pensi alle tariffe: la loro fissazione non doveva essere sensibile, a quel punto, agli indirizzi politici (e alle ragioni elettorali) dei governi, ma rappresentare il punto di equilibrio fra i costi delle imprese erogatrici, le ragioni degli utenti, i margini di incentivazione necessaria a stimolare la concorrenza via via che si veniva formando» (Amato 1996, 5). 
zi politico-amministrativi» (Amato 1996, 6). Lo scopo, cioè, è quella salvaguardia dei cosiddetti settori «sensibili» alle libertà dei cittadini mediante una loro tutela, «né propriamente amministrativa, né giudiziale» (Amato 1996, 6), dall'invadenza dei poteri pubblici e privati. Ecco dunque la Commission Nationale de l'Informatique e des Libertés, per tutelare la riservatezza dei cittadini nei confronti dei sistemi informatici automatizzati; la Commission des Opération de Bourse; la Commission d'Accés aux Documents Administratives; il Conséil de la Concurrence; il Conséil de l'Audiovisuel; la Commission de Sondages ${ }^{20}$. Autorità che taluni studiosi sogliono distinguere tra enti con poteri di regolazione e di decisione del caso concreto - come la commissione per l'informatica e le libertà e la commissione per le operazioni di borsa - e enti le cui funzioni si limitano ad attività di persuasione e di influenza ${ }^{21}$, ma che poggiano comunque sul presupposto della loro sottrazione alle direttive del governo, mentre la loro nomina registra l'ingerenza del Parlamento e/o la designazione di alte corti o di istanze professionali.

$\mathrm{Ma}$, a parte accenni sommari, un vero excursus comparatistico dovrebbe avere ben altra ampiezza di riferimenti e di argomentazioni, visto che di entità indipendenti troviamo esemplificazioni in tutto il mondo, anche se etichette simili celano differenze, spesso radicali, sul piano delle funzioni, dell'organizzazione interna e della loro interrelazione con $\mathrm{i}$ contesti economici, politici e istituzionali dei singoli paesi. E visto che non meno diverse sono le specifiche matrici ${ }^{22}$ e le contingenze storiche che hanno dato vita all'una o all'altra opzione istituzionale, ciò che preme sottolineare è come proprio questa varietà di circostanze e di razionalità genetiche ${ }^{23}$ riguardi anche il caso italiano. Anche se, in parallelo, possiamo rinvenire un minimo denominatore comune in cui sembrano connettersi gli elementi di tanta diversità.

20 Cfr. Morange (1987); Genevois (1989); Schrameck e Delcros (1988); Chevallier (1986); Maisl (1989); Sabourin (1989); Teitgen Colly (1989); Autin (1991).

21 Cfr. l'antologia curata da Colliard e Timsit (1989).

22 Poiché «i panorami nazionali sono pieni di contraddizioni, costruiti a pezzi e bocconi, ma presentano caratteristiche evidenti» (Cassese 1991, 383).

23 Per le quali cfr. ancora Majone e La Spina (1991); Diez Picazo (1997); D'Alberti (1995). 


\section{Una tipologia di riferimento}

Infatti, le multiformi esperienze nazionali evidenziano comunque la scelta di affidare la regolazione di fattori e relazioni importanti, spesso decisive, dell'osmosi tra poteri pubblici e processi di mercato - insieme alle contraddizioni e ai conflitti che vi si legano - ad istituzioni che possono, di diritto o di fatto, manifestare gradi e forme diverse di indipendenza dal sottosistema partiti-Parlamento-governo e dalla sua capacità di indirizzo e di vincolo sul merito delle decisioni inerenti ai relativi ambiti di regolazione. Istituzioni caratterizzate, allo scopo, da modalità d'azione regolativa - $\mathrm{i}$ cui procedimenti formali si innestano su intensi e non formalizzati processi di conflitto, confronto, comunicazione e negoziato sociale - autonome dagli apparati dell'amministrazione e da questi separate per legittimazione e responsabilità, mentre il decision making in cui si sostanzia la loro attività permane funzionalmente politico per capacità potestativa, di influenza e di interazione con i processi di policy e per l'impatto collettivo, ancorché indiretto, dei risultati del loro agire. Istituzioni, inoltre e per ciò stesso, affidate ad élites esenti (anche se di fatto non necessariamente immuni) da mandati politici di partito o dagli interessi in gioco nei singoli settori operativi e formalmente legittimate sulla base della loro specifica autorevolezza tecnico-cognitiva e professionale. Tali istituzioni, proprio da questa duplice estraneità, ricavano la loro missione e la loro legittimazione di regolatori «terzi» e super partes delle opportunità private, delle risorse collettive e dei processi allocativi. Il tutto con particolare riguardo a diritti e interessi che assumono, in determinate contingenze economiche, culturali e ideologiche, un peculiare rilievo per la sensibilità, la competizione o il conflitto sociale e che, come tali, si rivelano refrattari a un controllo politicamente partigiano o difficilmente funzionali al sostegno (specifico o diffuso, congiunturale o periodico) della classe politica e delle sue strategie contingenti di integrazione e di mobilitazione del consenso.

Abbiamo appena evocato le differenze rinvenibili nella molteplice gamma di authorities e di funzioni cui sono chiamate. $\mathrm{Da}$ caso a caso queste oscillano lungo un continuum che giustappone il polo di specifici poteri normativi, aggiudicativi e decisionali, tali da rendere le authorities $\mathrm{i}$ core actors dei rispettivi sottosistemi di interazioni, al polo rappresentato da particolari attività consultive, propositive o ispettive a supporto del con- 
trollo e della governabilità dei medesimi ad opera dell'autorità politica. Eppure, comunque si dislochino in questo ipotetico ventaglio di attività, le funzioni delle autorità indipendenti hanno pur sempre a che vedere con una qualche missione regolati$v a$, per quanto direttamente o implicitamente ascritta. Sappiamo anche - e sulla scorta di una sconfinata letteratura internazionale ${ }^{24}$ - che, più che una nozione, regolazione è un vero campo semantico, che può contenere l'intera gamma delle public policies ipotizzabili in capo al potere pubblico ${ }^{25}$. All'interno di tale campo si dipana quel grande ventaglio storico di esperienze politico-istituzionali di forme di controllo e di coordinamento pubblico degli interessi privati, che - tra le due sponde dell'Atlantico - si sono succedute, tra modelli variamente monistici o variamente pluralistici, per dotare il potere pubblico della capacità di controllare e influenzare le attività e la competizione degli interessi nel sistema economico e nel sistema sociale. Tuttavia, la regolazione cui mi riferisco e che mi sembra che l'esperienza italiana stia privilegiando, ha una propria parentela concettuale con formule dottrinarie che intendono per regolazione quell'attività del potere pubblico che «non solo restringe la gamma di attività di un soggetto, ma lo fa con strumenti di una istituzione politica che fonde in un unico apparato funzioni legislative, esecutive e giudiziarie» (Horwitz 1989, 46); o quel «processo che consiste nella restrizione intenzionale della gamma di attività di un soggetto da parte di un'entità non direttamente coinvolta nelle stesse» (Mitnick 1980, 9). E, soprattutto, quando ci riferiamo a quelle nozioni che, delle funzioni di regulation, privilegiano un peculiare profilo organizzativo e procedurale, vale a dire il suo consistere in «un controllo prolungato e focalizzato, esercitato da una agency pubblica, su una attività cui una comunità attribuisce una rilevanza sociale» (Selznick $1985,132)$ muovendo «dall'interno» della specifica arena di relazioni e conflitti in cui quella attività viene espletata. Una regolazione, insomma, come funzione di un potere pubblico che affida le sue ambizioni di governo non, o non solo, alla gestione o alla creazione diretta di attività di interesse collettivo, ma anche,

24 Per una accuratissima rassegna della quale cfr. Ferrari (1997, parte seconda).

25 È appena il caso di rammentare come Lowi $(1985,70)$ definisca «politica pubblica»: «una regola formulata da qualche autorità di governo che esprime l'intenzione di influenzare il comportamento dei cittadini, individualmente o collettivamente, attraverso l'uso di sanzioni positive o negative». 
o soprattutto, alla sua capacità di statuire regole (ivi comprese quelle di fonte legislativa o amministrativa o giurisprudenziale) e di darvi applicazione mediante agenzie, non propriamente amministrative né propriamente giurisdizionali, che operano autonomamente dall'autorità politica e amministrativa e dall'indirizzo di governo, e agiscono in «presa diretta» con le diverse sfere di interessi e con gli àmbiti materiali della loro azione. E lo fa privilegiando forme, tecniche e procedure di regolazione condizionale a detrimento o a integrazione, a seconda delle funzioni delle diverse agenzie, di modalità proprie della regolazione finalistica o di quella soltanto prudenziale ${ }^{26}$. Esemplare è in proposito la normazione antitrust: che persegue l'obiettivo di rendere correttamente funzionanti i mercati ponendo regole che attengono alla loro stessa struttura e che si pongono come «interne e coessenziali al concetto stesso di mercato» (Clarich 1993, 121). Mentre il ricorso a discipline amministrative di settore (ad esempio mediante la previsione di prezzi amministrati nei confronti di monopoli naturali, oppure mediante la statuizione di regole amministrative e di apparati pubblici preposti al controllo delle imprese bancarie e assicurative), o a forme diverse di impresa pubblica (nazionalizzata o controllata altrimenti dallo Stato) si pone invece come correttivo esterno alle regole di mercato ${ }^{27}$.

26 «La regolazione condizionale può essere sommariamente definita come un insieme di regole che al contrario di quanto avviene per le regole di tipo finalistico e prudenziale, non prefigura un risultato o un obiettivo al quale l'attività privata deve conformarsi, bensì contiene criteri di comportamento e regole di condotta rivolti agli stessi privati», mentre la regolazione finalistica si compone «di comandi specifici» ai quali seguirà «un'attività di controllo volta ad accertare l'uniformità fra comando e sua esecuzione da parte dei soggetti coinvolti» entro uno schema «basato sulla contrapposizione fra interesse pubblico e situazioni soggettive private: per la cura del primo, rimesso all'apprezzamento discrezionale dell'autorità, le seconde sono soggette a comandi e controlli». Quanto alla regolazione di tipo prudenziale, questa si distingue dalla precedente in quanto «mentre nella regolazione finalistica sono individuate a priori le condizioni di conformità dell'attività privata all'interesse pubblico, qui l'attività amministrativa è volta a comporre diversi interessi: la cura concreta dell'interesse pubblico risulterà, secondo la nota formula, dal rapporto fra situazioni soggettive diverse, potenzialmente contrapposte»; ove, invece, nel caso della regolazione condizionale «criteri e regole (...) non trovano fondamento nella garanzia o nella tutela di un interesse pubblico, ma, piuttosto, nella necessità ed opportunità di garantire e tutelare, reciprocamente, situazioni soggettive private: di qui (...) la possibilità di configurare un interesse pubblico "riflesso" e mediato, affidato, appunto, alla cura delle autorità indipendenti» (Torchia 1996, 56-58).

27 Rispetto al quale, a una regolazione condizionale non si chiede «di apprezzare e ponderare gli interessi, ma, invece, di verificare e assicurare che fra essi si mantenga un determinato equilibrio: la libertà di eguale accesso al mercato per gli operatori, la liber- 
In questa chiave, è possibile restringere sensibilmente l'area semantica della regolazione e porre l'accento sulle dimensioni più qualificanti del modello che vi si può rinvenire. Gli elementi più salienti, ai fini del caso italiano, mi sembrano articolabili nei termini seguenti.

1. Le funzioni di regolazione concernono attività o posizioni private che sono rilevanti ai fini della (o delle) collettività, e che riescono a venir controllate, indirizzate ed eventualmente modificate, così come tutelate e sostenute, mediante agenzie autonome dall'apparato amministrativo dello Stato e tendenzialmente indipendenti dall'indirizzo delle istituzioni politiche, in quanto la loro importanza e il grado di conflittualità economica, o di sensibilità sociale o culturale che le connota intrinsecamente, le rende, nel breve o nel lungo termine (secondo i casi), difficilmente compatibili con cicli politici - elettorali o coalizionali - di governo o difficilmente componibili entro strategie rappresentative o negoziali di partito.

2. Ne consegue che l'attività regolatrice si avvarrà, più che di strumenti autoritativi del genere commands and controls, di modalità di interazione con $\mathrm{i}$ soggetti interessati miranti a sollecitarne il coinvolgimento e la collaborazione, mediante strumentazioni che sappiano combinare premi, incentivi e coazione.

3. Le agenzie ad boc da cui dipendono l'effettività e l'efficacia del «controllo prolungato e focalizzato» sono specificamente attrezzate a che, lungi dall'una tantum provvedimentale della voluntas legislativa, alimentino e guidino un processo continuativo di osservazione dell'attività da regolare, valutino e confrontino gli interessi in gioco mediante specifiche procedure cognitive, dibattimentali e probatorie, e provvedano alla revisione o alla riformulazione delle regole in funzione delle circostanze e del loro divenire o, quanto meno, alla loro sollecitazione e progettazione.

4. La legislazione specifica con cui l'autorità politica «delega» alla singola istituenda agenzia regolativa le relative competenze, si limita a delinearne i principî ispiratori e le finalità essenziali, a fronte di materie, processi e rapporti la cui regolazione implica grande flessibilità definitoria e interpretativa. Ne deriva che le strategie e le agende della regolazione, insieme alla 
definizione delle sue modalità processuali, rimangono largamente affidate all'autonoma sensibilità politica e istituzionale e al profilo etico, culturale e comunicativo dei «riconosciuti» esperti che quelle agenzie vengono a reggere. In una parola, alla loro capacità di muoversi stabilmente «dentro» l'insieme delle reti intersoggettive che formano il singolo subsistema di interessi, od almeno di acquisirne piena e continuativa cognizione attraverso l'osservazione «introspettiva» del loro interagire, pur manifestandosi da essi indipendenti nella propria attività valutativa ed arbitrale.

5. Per quanto in misura variabile ed empiricamente problematica (poiché da osservarsi «all'opera» e non solo né tanto sulla scorta delle regole che presiedono alla selezione dei responsabili), l'indipendenza delle «autorità indipendenti» nell'esercizio delle loro funzioni, è - per definizione - il loro stesso postulato esistenziale e legittimante. L'ovvia conseguenza è che le agenzie non possono comunque avvalersi del riflesso della legittimazione dell'autorità politica per confortare la propria autorevolezza di policy makers. Questa, al contrario, debbono conquistarsela sul campo, dando testimonianze salienti della propria autonomia di giudizio insieme alla competenza professionale e al prestigio personale nel cui nome i componenti le singole autorità divengono tali. Tuttavia, il grado di consensualità che essi sanno imprimere ai processi decisionali che presidiano e la loro connessa capacità di egemonia nella rete di relazioni in cui sono chiamati ad operare, possono certo alimentarne un'autonoma legittimazione.

Questi elementi più generali di un possibile modello rimarcano anche come la legislazione che legittima l'azione di tali agenzie regolative, esprima certo la percezione (non importa quanto condivisa) da parte dell'autorità politica della «crescente insofferenza verso la politica delle democrazie contemporanee» (Amato 1996, 16) e delle ragioni «di un ridimensionamento della sfera che le è riconosciuta: la sfera, di sicuro, delle decisioni di redistribuzione fra categorie sociali diverse» (ibidem) non quella delle decisioni che, toccando diritti e funzioni predefinite della cittadinanza, «devono essere al riparo dagli indirizzi e dalle esigenze elettorali della politica» (ibidem). Ma, nei moventi dichiarati o facilmente rinvenibili nei propri processi legislativi, è anche una normazione che enfatizza - in luogo delle logiche, delle aspettative e delle immagini dello Stato distributivo e soprattutto dello Stato interventista e pianificatore - una conce- 
zione del potere pubblico che poco crede ai programmi di lungo termine e alla loro realizzabilità operativa, alle possibilità di dare soddisfazione alla globalità della domanda sociale, a una gestione complessiva e sinottica dello sviluppo economico, e all'efficacia delle proprie capacità di darvi diretta ed esaustiva regolazione. Una concezione dello Stato, cioè, chiamato a «fornire risposte specifiche a problemi circoscritti, rispettando, per quanto possibile, le logiche d'azione dei sistemi regolati» (Majone e La Spina 1991, 22) e che - in una chiave radicalmente antirousseauiana e contrapposta alla tradizione giuspositivistica che lo vuole parametro aprioristico e autoritativo - pare privilegiare una nozione di pubblico e generale interesse quale risultato incrementale di un mutuo ma controllato aggiustamento entro il particolarismo degli interessi che si confrontano e competono in una determinata arena. Una visione dello Stato, infine, che non abdica alla sua missione regolatoria ma ne demanda l'esercizio al di fuori della sfera dell'autorità politica, affidando le (formalmente) «sue» conseguenti politiche regolative ad arene esterne, di cui si limita a garantire la pubblica legittimità, dotandole delle condizioni e delle risorse legali e organizzative per le interazioni che vi hanno luogo.

\section{Gli estremi della vicenda italiana}

Come si vede, regolazione e indipendenza sono le due parole chiave alla base del modello più generale. Si tratta ora di vedere come esse siano state declinate nel caso italiano. Il loro diverso combinarsi ha dato luogo a una pluralità di modelli specifici, che a loro volta derivano da una pluralità di circostanze storiche su cui si sono innestate differenti e peculiari motivazioni e quindi una pluralità di soluzioni organizzative e funzionali, che solo in una ricostruzione ex post consentono di individuare filamenti e denominatori comuni.

Se si fa eccezione per la Banca d'Italia, che risale a circostanze e a logiche politico-istituzionali specifiche, e che dispone di caratteri originari non contenibili entro il contesto di autorità indipendenti a noi più prossimo (seguendo un'evoluzione che ne ha via via allargato la sfera di indipendenza, che dall'assorbimento in sé dell'originaria funzione di vigilanza bancaria di cui era delegataria dallo Stato, l'ha resa attualmente l'autorità di tutela della concorrenza nel settore bancario, fino a farne il caso 
più intenso e radicale di autorità indipendente nel panorama italiano $)^{28}$, il processo di attivazione delle autborities italiane si è articolato in scansioni precise nel corso dell'ultimo ventennio.

Una prima scansione coincide con l'istituzione della Consob, commissione nazionale per le società e la borsa, creata nel 1974 al fine di vigilare sulla trasparenza del mercato azionario. All'origine del nuovo organismo possiamo individuare motivi molto contingenti, tra i quali spicca l'esigenza di prevenire un conflitto interburocratico concernente la ripartizione e la convivenza di più competenze ministeriali circa il funzionamento del mercato mobiliare. Di qui l'opportunità di una soluzione che prevenisse un simile contenzioso mediante la creazione ex novo di un organismo ad boc, dotato di una parziale indipendenza (data la sua soggezione alla vigilanza dell'amministrazione ratione materiae più forte, quella del tesoro), ove venissero concentrate competenze prima distribuite fra più amministrazioni. Di qui l'idea di un collegio di nomina governativa, sottoposto al parere non vincolante delle commissioni parlamentari, suscettibile di scioglimento per impossibilità di funzionamento, con limitata autonomia organizzativa e una capacità decisionale giuridicamente efficace solo mediante decreti del ministro del tesoro. Una corposa evoluzione legislativa ne avrebbe poi allargato l'autonomia, sino a consentirne attualmente la configurazione entro il novero delle autorità indipendenti, anche se, a tutt'oggi, alcuni suoi atti devono assumere la forma o del decreto del capo del governo o del ministro.

Occorre però arrivare agli anni novanta perché il fenomeno

$28 \mathrm{E}$ al quale ben si attaglia quanto dirà il Tribunale costituzionale tedesco in un'importantissima sentenza del 12 ottobre 1993 commentando la posizione del Sebc, quale risulta dall'art. 105 del trattato di Maastricht. In essa viene affermato che «un settore politico essenziale, che tutela con il valore della moneta la libertà individuale e condiziona con il volume della moneta anche la finanza pubblica e le politiche che ne dipendono, viene sottratto al potere di indirizzo dei titolari dei poteri sovrani e - salva una revisione del Trattato - anche all'intervento del legislatore sui relativi compiti e strumenti in azione. Rendere autonoma la maggior parte delle funzioni connesse alla politica monetaria attribuendole ad una banca centrale indipendente significa svincolare un potere pubblico sovrano dalla diretta responsabilità parlamentare a livello statale o sovra-nazionale, al fine di sottrarre la moneta al dominio di gruppi di interesse o di rappresentanti politici interessati alla rielezione». Nella stessa sentenza si asserisce che «l'autonomo affidamento della politica monetaria alla competenza sovrana di una Banca centrale europea indipendente - secondo un modello non trasferibile ad altri settori politici - rispetta i limiti costituzionali entro i quali può essere modificato il principio democratico». Per il testo della sentenza vedi «Giurisprudenza costituzionale», 1994, II, 677 ss., qui 706, cit. da Predieri (1997a). 
delle autorità indipendenti assuma quell'evidenza e quella pregnanza politico-istituzionale che lo rendono un elemento imprescindibile del panorama italiano e della lunga transizione del sistema politico di questo decennio. $\grave{E}$ un processo istitutivo molto intenso e concentrato nel tempo: tra il giugno e il settembre del 1990 il legislatore vara la commissione di garanzia per l'esercizio del diritto di sciopero nei servizi pubblici essenziali, il garante per la radiodiffusione e l'editoria, l'autorità per la concorrenza.

Il primo organismo (istituito con la legge 12 giugno 1990, $n$. 146) è quello a indipendenza più tenue dei tre. E il risultato del «difficile compromesso tra le ragioni degli utenti e quelle dei sindacati» (Amato 1996, 7) del lavoro dipendente nel settore pubblico. La soluzione escogitata consiste nella limitazione (essenzialmente procedurale) del diritto di sciopero in cambio della non ingerenza dell'Esecutivo nel suo esercizio. Di qui la previsione di una apposita agenzia di mediazione e raccomandazione chiamata ad operare «in un difficile equilibrio tra diritti costituzionalmente diversi» (Amato 1996, 8), mediante un'attività di negoziazione che trae legittimazione da una normazione legislativa di principio, e investitura non dal governo ma dal Capo dello Stato su diretta ed esclusiva designazione dei presidenti delle Camere: con «una procedura, rispetto alla tradizione, singolarissima, che trasporta al di fuori dei lavori parlamentari, utilizzandolo nell'ordinamento generale dello Stato, quel principio di neutrale imparzialità che i Presidenti delle Camere avevano in precedenza incarnato soltanto intra moenia» (ibidem).

La seconda autorità (il garante per la radiodiffusione e l'editoria) nasce a distanza di pochissimi mesi, con la legge 6 agosto 1990, n. 223. Nominato dal Capo dello Stato su proposta adottata d'intesa dai due presidenti delle Camere, il garante ha poteri normativi, di vigilanza e di sanzione sulle utenze radiofoniche e televisive, e diventa poi anche autorità di garanzia della concorrenza nel settore dei mezzi di comunicazione di massa, oltre che della «par condicio» nella loro utilizzazione elettorale. «Il Garante ha il massimo dei connotati dell'indipendenza e li ha come sbocco naturale di una lunga storia, che è del tutto peculiare: la storia della televisione in Italia, nata come monopolio pubblico, difesa in quanto tale dalla Corte costituzionale purché venisse sottratta all'Esecutivo, trasferita per ciò stesso «nell'area del Parlamento» e per questa ragione già dagli anni settanta innestata in una cornice istituzionale che escludeva ap- 
punto il governo. Risalgono ad allora l'attribuzione al Parlamento della elezione del Consiglio di amministrazione della Rai e della vigilanza sul suo funzionamento. Quando arriveranno le televisioni private e si porrà la questione della vigilanza sull'insieme del sistema misto, sarà naturale e consequenziale la sua attribuzione ad un organo del tutto indipendente dall'Esecutivo e di derivazione solo parlamentare» (Amato 1996, 8). Anche se il garante, per profili essenziali come la fissazione annuale dei limiti pubblicitari della concessionaria pubblica del servizio radiotelevisivo, è solo organo consultivo del governo, mentre per altri, ha solo poteri di impulso verso l'Esecutivo, come per l'irrogazione di sanzioni di competenza del ministro delle poste e delle comunicazioni ${ }^{29}$. Inoltre, per meglio prevenire le propensioni lottizzatorie connesse alla rilevanza per la competizione politica dello stesso settore di cui il garante avrebbe dovuto occuparsi, è stata scelta la soluzione dell'organo monocratico ${ }^{30}$.

La terza autorità, che contraddistingue questo momento fondativo degli inizi del decennio in corso, viene istituita con la legge generale sulla concorrenza, la n. 287 del 10 ottobre 1990. Diversa per ratio e moventi istitutivi rispetto al garante per la radiodiffusione e l'editoria, l'autorità per la concorrenza e il mercato, nonostante la sua collegialità che la differenzia dalla monocraticità del primo, ne ricalca però il grado e le ragioni di indipendenza, in stretto parallelismo. Tuttavia, la genesi dell'antitrust italiana ha radici più antiche di quanto non evidenzino le sincronie costitutive rispetto al garante, e che risalgono alla pluridecennale refrattarietà del legislatore italiano, a differenza degli altri paesi fondatori, ad adeguarsi alle disposizioni comunitarie che già nel Trattato di Roma vietavano, per l'appunto, l'adozione di intese restrittive della concorrenza e gli abusi di posizioni dominanti sui mercati della Comunità. Non che la nostra classe politica non si fosse cimentata nella materia, né che fossero mancate iniziative legislative anche autorevoli ${ }^{31}$, insieme a un dibattito culturale di tutto rispetto (Peluffo 1996): ma si era (1995)

29 Cfr., per i profili tecnici, Roppo e Zaccaria (1991); Falcon (1993); Caretti

30 «(...) anch'esso, tuttavia, «catturabile», e certamente meno in grado, rispetto a una pluralità di commissari, di controllare un settore così complesso» (La Spina 1996, 297).

31 Cfr., in particolare, le proposte di legge Malagodi e Bozzi del 16 marzo 1955 (C.n. 1521); Lombardi e La Malfa del 12 settembre 1958 (C.n. 248); e Amendola e altri del 15 gennaio 1960 (C.n. 1903). 
trattato di una breve fiammata, destinata a spegnersi dopo i primi anni sessanta. D'altra parte, la cultura economica e istituzionale italiana «era lontana le mille miglia dalla concorrenza e, influenzati ancora dalle nostre origini di paese a sviluppo industriale ritardato, affidavamo il nostro sviluppo non ad essa, ma alle imprese pubbliche, agli aiuti di Stato, ai diritti di esclusiva e ad altre penetranti forme di regolazione pubblica» (Amato $1996,9)$. Ben prima che la crisi della politica italiana desse una spallata decisiva alle argillose fondamenta della nostra partitocrazia e delle sue élites, sarebbero stati il corposo impulso comunitario e la pervasività del grande mercato europeo ad agitare - con le proprie reiterate sollecitazioni - un legislatore recalcitrante $^{32}$, dopo l'approvazione dell'Atto unico del 1986 e l'obbligo del completamento conseguente l'integrazione del mercato comune entro il 1992, costringendolo a porre finalmente mano a una normazione antitrust ${ }^{33}$ e a rimuovere i suoi antichi dilemmi ${ }^{34}$. Tant'è che la legge del 1990 viene varata dopo un processo legislativo privo di particolari asperità: con i partiti della maggioranza che sostengono in modo abbastanza acritico il progetto governativo, e con le forze d'opposizione di sinistra che si mantengono su una posizione «costruttiva» e «responsabile», pur con talune increspature circa gli effetti protettivi che

32 Prendo a prestito da Predieri (1987) il titolo di uno dei suoi volumi che più compendia le vischiosità del processo legislativo italiano nei confronti della normazione comunitaria.

33 Circa le fasi propedeutiche del processo legislativo della legge generale sulla concorrenza (dalla bozza di «codice dell'impresa» della commissione Ferri del 1983, al rapporto della commissione Romani del 1988, prima per impulso del ministro dell'industria Zanone e poi del suo successore Battaglia, al programma di governo del ministero De Mita pur in assenza di specifiche sollecitazioni dei partiti che ne formavano la coalizione, poi sostituito dal governo Andreotti dopo la crisi del luglio del 1989, al progetto di Guido Rossi, al disegno di legge Battaglia che accoglieva quasi totalmente le linee del rapporto Romani e che costituì la base della legge 287 del 1990) e il suo svolgersi, cfr. Delle Cave (1994) e anche Sacconi (1992).

34 Tra questi il più corposo aveva a suo tempo riguardato, per l'appunto, l'autorità da preporre alla tutela della concorrenza, cui affidare la valutazione di intese restrittive, concentrazioni e abusi, e le correlative decisioni. All'epoca delle proposte sopra menzionate, la discussione verteva, in particolare, sui poteri decisionali o soltanto istruttori che avrebbero dovuto connotare la nuova autorità. Si trattava di stabilire se essa avesse dovuto rivestire un ruolo solo istruttorio, restando al ministero dell'industria il compito della decisione, il che era del tutto coerente con la visione e l'auspicio di un'economia da affidare ad una missione governativa direttamente e specificamente responsabile per le sue dinamiche e per le connesse politiche industriali; o se l'autorità indipendente avesse dovuto venir dotata di entrambi i tipi di funzioni, anche se con poteri più istruttorî che decisorî. 
la legge avrebbe potuto sortire a favore delle maggiori industrie nazionali e circa l'estensione dell'autonomia decisionale della nuova autorità. Se mai, preoccupazioni più corpose si sono manifestate da parte della Banca d'Italia, timorosa di perdere le proprie potestà sul sistema creditizio e sul suo controllo. Tuttavia, alla legge si è appunto arrivati in modo relativamente agevole. Il risultato, per quanto attiene alla authority così istituita, è un organismo collegiale di cinque membri, tutti nominati direttamente dai due presidenti delle Camere, che diventano, pertanto, non solo i titolari della designazione, ma i responsabili esclusivi dell'investitura, senza che altro organo costituzionale ne debba sancire le scelte né partecipare alla loro formazione. La nuova autorità viene poi dotata di una compiuta autonomia organizzativa e contabile, e vede garantita la completa estraneità dell'Esecutivo (salve le ipotesi eccezionali delle concentrazioni di interesse nazionale) dalle sue decisioni, suscettibili del solo sindacato giudiziario (che compete in esclusiva al tribunale amministrativo del Lazio).

La terza scansione istitutiva delle authorities italiane coincide con il pieno dispiegarsi della transizione dal regime partitocratico della prima repubblica verso i nuovi ed incerti assetti maggioritari del sistema politico, e con l'aggiornamento di autorità organizzativamente esterne $\mathrm{ma}$ non estranee al raggio d'azione dell'Esecutivo (Isvap, commissione per l'accesso ai documenti amministrativi, autorità per l'informatica nella pubblica amministrazione, commissione di vigilanza sui fondi pensione, autorità portuali, autorità per la vigilanza sui lavori pubblici, agenzia nazionale per la protezione dell'ambiente, agenzia per i servizi sanitari regionali, agenzia per la rappresentanza sindacale delle pubbliche amministrazioni). Una diffusione di enti regolatori e di controllo che trova la sua manifestazione più emblematica nell' 'istituzione delle autorità di regolamentazione dei servizi di pubblica utilità in correlazione alla loro privatizzazione, ad opera della legge 481 del 14 novembre 1995, e nella disciplina generale delle ulteriori autorità da costituire successivamente in tale medesima prospettiva (la legge ha disposto, infatti, l'immediata messa in opera delle sole autorità concernenti l'energia elettrica e il gas, rinviando quella per le telecomunicazioni pur disciplinata nei «principî generali» - a un «definitivo» riassetto del settore).

Il processo di privatizzazione di questi servizi è appunto la molla essenziale della scelta del legislatore di ristrutturare la re- 
golazione del settore mediante una rete di authorities regolative. Le motivazioni sono sostanzialmente analoghe a quelle accennate a proposito dell'esperienza inglese: sostenere la transizione da monopoli od oligopoli pubblici, o pubblicisticamente garantiti, operanti nell'area dei servizi di pubblica utilità, verso specifici mercati competitivi, mediante una regolazione dipendente non più dagli indirizzi politici (e dalle preoccupazioni elettorali o di competizione partigiana) dei governi e dalla discrezionalità degli apparati burocratici competenti, ma dalla relazione funzionale tra strategie autonome di impresa, aspettative di qualità e di costo dei servizi effettivamente tutelabili per gli utenti, concrete stimolazioni e incentivazioni della concorrenza tra le aziende. Esigenze la cui soddisfazione le autorità settoriali dovrebbero promuovere e assicurare attraverso un'articolata gamma di funzioni di regolazione di natura «prudenziale» ${ }^{35}$, proponendosi, rispetto ad esse, come una scelta istituzionale quasi fisiologica.

La loro indipendenza non è «totale», ma è funzionale alla intensa pregnanza pubblica dei beni e delle prestazioni che sono oggetto delle attività privatizzate e alla conseguente generale «responsività» politica che ne deriva in capo all'autorità di governo e, sullo sfondo, a quella parlamentare: poiché «se taluni servizi pubblici sono passati dalla mano pubblica a quella privata, lo Stato non se ne disinteressa e i servizi, per esser gestiti da privati, non divengono meno pubblici» (Cassese 1991, 385). Così, mentre l'autonomia organizzativa e contabile delle autorità è analoga a quelle dotate di massima indipendenza, il congegno di nomina dei membri dei loro collegi è articolato in una designazione del ministro, settorialmente competente, al Consiglio dei ministri, che la fa propria con apposito decreto, previo il placet delle commissioni parlamentari di merito, espresso a maggioranza dei due terzi dei loro componenti ${ }^{36}$. Né è del tutto indipendente l'esercizio dei poteri assegnati che, per taluni profili essenziali (come il rilascio delle concessioni alle imprese), si coniuga con quelli del governo entro procedimenti unitari in cui è a quest'ultimo che compete la parola conclusiva.

35 Cfr. la nota 26 che precede.

36 Ed è un parere che presuppone convinte motivazioni, visto che è prevista la possibilità per le commissioni di procedere all'audizione delle persone designate a comporre il collegio dell'autorità istituenda (art. 2, comma 7 della legge 481/1995). 


\section{Un possibile modello per le authorities italiane degli anni novanta}

Abbiamó ripetuto, e appena visto per il caso italiano, come le autorità indipendenti non diano vita ad un modello unitario. Dietro il nomen che le accomuna, c'è una pluralità di circostanze storiche e di moventi differenziati di policy, che danno vita a istituti diversi per funzioni e per relazioni con i poteri dello Stato e con i preesistenti apparati di governo e dell'amministrazione.

Una classificazione della varietà delle autborities sotto tale duplice profilo (quali funzioni e quali relazioni o interazioni con i diversi specifici ambienti istituzionali e amministrativi) fuoriesce dal contesto di questi appunti. È certo che chi volesse cimentarvisi dovrebbe fare i conti con una gamma estesa di variabili da ponderare. Così, ad esempio, si dovrebbe valutare come si collocano e quanto si integrano le loro funzioni nei singoli subsistemi che le autorità sono chiamate a regolare ${ }^{37}$. Oppure andrebbero ponderati il raggio d'azione e la capacità regolatoria delle loro funzioni: distinguendo tra autorità le cui funzioni investono un ambito vasto ma definito di interessi o di ruoli economici e sociali $i^{38}$, o una pluralità indefinita dei medesi$\mathrm{mi}^{39}$. E occorrerebbe discriminare tra autorità che «decidono» $\mathrm{e}$ autorità che «consigliano», «propongono», «indagano», «per-

37 Se cioè si tratta di autorità disposte al vertice di quel sottosistema, con poteri amministrativi che si sommano o meno ad autonomi poteri di intrapresa economica (come nel caso della Banca d'Italia), o se si tratta di autorità preposte alla regolazione di quel sottosistema rispetto alla cui dinamica si pongono come noduli necessari e integrati nel reticolo delle loro interazioni decisionali, ma non come operatori autonomi nei medesimi. Cfr. Predieri (1997a, 51 ss.) il quale sottolinea come tali autorità-nodulo (che egli esemplifica nella Consob e nel Garante) rimangano «esterni al subsistema in quanto non operatori economici in quel segmento di mercato, ma contemporaneamente, sono immersi in quel singolo subsistema. Con il quale hanno uno scambio fittissimo e continuo, non limitato agli atti e ai procedimenti formali, arricchiti o meno da consultazioni, in un intreccio continuo di informazioni, e con il rischio di negoziazioni, che possono investire provvedimenti di dettaglio, ma anche strategie». Insomma, «i noduli, prima o poi, in taluni momenti possono identificarsi con il subsistema» in una sorta di «autoregolazione, in cui c'è il pericolo del prevalere degli interessi più forti del settore, che possono portare a spartizioni di potere di sapore oligopolistico, riportandosi, al di là delle forme, a sostanze di oligopoli amministrati che per anni hanno caratterizzato la nostra economia» (ibidem). mercati.

38 Come avviene per le autorità preposte ai servizi di pubblica utilità e ai relativi

39 Come avviene, invece, per l'autorità garante della concorrenza e del mercato, della cui globalità di interazioni è appunto affidataria, o per la commissione per l'esercizio del diritto di sciopero nei servizi pubblici. 
suadono ${ }^{40}$; autorità che «controllano» e «indirizzano» e autorità che controllano ma non indirizzano ${ }^{41}$; autorità, che pongono regole e autorità che attuano regole; autorità che incidono direttamente in un certo insieme di interazioni, con una propria capacità di indirizzo o di comando, e autorità che possono solo influenzare determinate dinamiche. Così come andrebbero considerate le modalità decisionali delle singole autorità: distinguendo quelle autorità la cui attività regolatoria contempla determinate procedure e garanzie di contraddittorio a favore degli interessi in gioco, da quelle la cui interazione con i medesimi resta affidata solo a virtuali modalità informali di confronto e di dialogo. Oppure, ancora, si dovrebbe valutare il grado di innovatività istituzionale e funzionale delle autorità, discriminandole a seconda che siano assegnatarie di funzioni già esercitate da altri organi o enti dell'amministrazione pubblica e poi ridefinite entro un nuovo modello organizzativo ${ }^{42}$, od invece esercitino funzioni prima inespresse dal potere pubblico ${ }^{43}$. Oppure, e soprattutto, occorrerebbe misurare il grado di indipendenza delle diverse autorità e osservare come esso si correli alle altre variabili in gioco. E, a tale scopo, bisognerebbe disporre di indicatori sintetici sufficientemente maneggevoli, in cui coniugare i profili formali della nozione di indipendenza con quelli che ne sono derivazione diretta e indotta. Indicatori che dovrebbero combinare profili tecnici e profili esistenziali. Una cosa, infatti, è l'indipendenza generalmente concepibile «come non soggezio-

40 Tra le quali potremmo collocare la commissione sull'esercizio del diritto di sciopero nei servizi pubblici essenziali, istituita dalla legge 146 del 1990, «che è autorità di tutela e garanzia dei diritti della persona, non di regole di mercato, con funzioni di mediazione, istituzionalmente tale (...), di garanzia in senso stretto, di persuasione morale, di contemperamento di interessi, chiamata a valutare atti, che funge da catalizzatore, interagisce con le parti, favorisce l'emersione degli interessi e delle esigenze da soddisfare, governa la sperimentazione degli assetti di volta in volta raggiunti, prepara il loro superamento, elabora gli standards, li applica con una attività complessiva e ampia e può esprimere un lodo sul merito del conflitto sindacale» (Predieri 1997a, 112).

41 Come nel caso della Consob che, pur dotata di poteri autoritativi nella regolazione del mercato di borsa e che possono portare fino alla sua chiusura sulla base di una valutazione del tutto discrezionale, «controlla non già con un controllo-direzione, ma con un controllo-verifica, (...) la produzione di informazioni imposta dalla legge (ad esempio nel caso di bilanci o di relazioni) e la loro diffusione o «socializzazione», e in taluni casi la impone, (...) con esclusione di controlli-direzione sulla gestione delle imprese» (Predieri 1997a, 109).

42 Come nel caso dell'Isvap, anche se la sua sostituzione di preesistenti apparati e funzioni governative non può dirsi compiuta (Costi 1995).

43 Come, ad esempio, nel caso dell'autorità antitrust ed in quello del garante per la radiodiffusione e l'editoria. 
ne al potere di indirizzo politico del governo-Parlamento e all'indirizzo amministrativo del governo o di singoli Ministri» (Massera 1988, 452) e che, per essere tale, deve tradursi in specifiche e riservate competenze decisionali o arbitrali in capo alle autorità, e nell'inesistenza di altrui poteri di emanazione di ordini, direttive e atti di indirizzo e coordinamento che ne vincolino le autonome capacità di valutazione e decisione ${ }^{44}$. Altra cosa è un'indipendenza che, oltre che in un'autonomia autorganizzativa, contabile, di autoindirizzo e di autolimitazione, possa anche tradursi in capacità di vincolo o di condizionamento dell'attività di terzi. Altra cosa ancora è una capacità specifica di indirizzo verso gli altri attori del singolo subsistema di interessi, immune da omologhi atti di indirizzo di altri enti o organi dell'apparato di governo, dell'amministrazione o del Parlamento ${ }^{45}$. E cosa ancora diversa, infine, è parlare di indipendenza con riferimento all'effettività di logiche interpretative e di stili decisionali con cui la singola autorità dia attuazione alle norme legislative, costituzionali o comunitarie alla cui applicazione è chiamata, nel dirimere i conflitti che si agitano nella sfera degli interessi cui sovraintende.

Inoltre, in qualunque ipotesi classificatoria, le autorità possono annoverare - nel loro formato funzionale e interattivo molteplici modalità di ciascuna di quelle variabili o di altre ancora ipotizzabili. Se infatti le autorità sono molte e diverse sono le loro funzioni, sono molteplici anche le «caselle» in cui ciascuna di esse può risultare simultaneamente inquadrabile. E se poi l'analisi volesse staccarsi dalla configurazione prescrittiva e addentrarsi nelle esperienze effettuali, e cogliervi tendenze e periodizzazioni di messa in opera, il quadro non potrebbe non risultarne ancora maggiormente articolato e complicato. Tanto più se teniamo conto di come, attorno al nocciolo normativo che ne disciplina le attività, le funzioni delle autorità indipendenti siano destinate a produrre «aloni» di effetti e di funziona-

44 «Altro è un ente o un'autorità che si indirizza, altro è un'autorità che è indirizzata, altro è garantire la concorrenza con guidelines del governo, altro con direttrici della autorità amministrativa indipendente» (Predieri 1997a, 86).

45 Così, abbiamo «autorità che si indirizzano e indirizzano, come la Banca d'Italia, autorità che si indirizzano e non indirizzano, come la Consob o l'Autorità garante della concorrenza e del mercato, autorità che vengono indirizzate (se le consideriamo indipendenti o quasi indipendenti) e indirizzano, come l'Agenzia per l'ambiente, e autorità che vengono indirizzate (se le consideriamo, anche in questo caso, indipendenti o quasi indipendenti) e non indirizzano, come l'Isvap» (Predieri 1997a, 92). 
lità non prescritte ma egualmente correlate, e che investono l'azione di altri organi politici e amministrativi dello Stato così come dei sistemi di interessi correlati (Predieri 1997a, 53 ss.). Aloni di effetti e di funzionalità che soli possono dare una misura realistica del rendimento di questi istituti, ben al di là degli output in cui le loro competenze, normative o/e provvedimentali o/e mediatorie, possano esprimersi.

Ciononostante, si può forse cogliere, almeno con riferimento alle autorità di garanzia create nel 1990 (garante per l'editoria e la radiotelevisione, antitrust, commissione per il diritto di sciopero, insieme all'antesignana Consob) e in relazione alle autorità chiamate a regolare la gestione dei servizi di pubblica utilità create nell'ultimo periodo, la logica di un modello tendenziale, in cui il legislatore sembra voler inquadrare le autorità indipendenti italiane e $\mathrm{i}$ rapporti in cui si pongono con $\mathrm{i}$ rispettivi ambienti istituzionali e amministrativi. Un modello che potrebbe fondarsi proprio sul «bipolarismo» tra la prima e la seconda specie di autorità. E cioè, quando il loro fine costitutivo contempla funzioni, pretese e aspettative private che si qualificano come diritti costituzionalmente tutelati nella titolarità di pari opportunità e di pari condizioni con cui confrontarsi o competere, allora le funzioni cui sono chiamate le autorità competenti sono appunto di garanzia e di arbitrato. Non trattano il nocciolo sostantivo dei problemi che affrontano, né danno risposte specifiche e concrete a bisogni o domande insoddisfatte. Non entrano nel merito del gioco, ma ne pongono o interpretano le regole o ne controllano l'applicazione. Di qui la loro separazione dal governo e dall'amministrazione dello Stato, sia per le nomine (solo di matrice parlamentare) che per le funzioni, conferite in esclusiva ed esercitabili senza interferenze governative. Quando il fine, invece, attiene ad una serie di interessi (che diritti non sono ma solo interessi rimangono) che l'autorità è chiamata a ponderare mediante opzioni che entrano concretamente e tecnicamente nel merito del gioco (fissando, ad esempio, i parametri delle tariffe dei servizi pubblici in concessione) e delle decisioni sostantive e finali, per comporre le aspettative e gli eventuali conflitti che vi si legano - «l'aspettativa degli utenti ad un servizio di buona qualità ad un prezzo ragionevole, le ragioni dell'economia aziendale, l'equilibrio nella destinazione degli utili fra distribuzioni presenti e investimenti futuri» (Amato 1996, 14), e via dicendo -, ecco allora che le nomine dei responsabili delle authorities tornano ad essere non più solo 
parlamentari, ma anche governative oltre che parlamentari. Ed ecco anche che le funzioni delle autorità, quanto a titolarità e ad esercizio, assumono forme di condivisione con organi del governo e dell'amministrazione statale.

La logica unitaria del modello pare evidente. Nel secondo caso, abbiamo autorità che sostanzialmente razionalizzano e articolano l'agire amministrativo in funzione di interessi affidati alla duttilità e alla competenza cognitiva e negoziale di organismi autonomi, ma che restano qualitativamente ascrivibili alla ponderazione discrezionale della pubblica amministrazione sulla base degli indirizzi politici di governo e Parlamento. Solo che quei compiti di ponderazione li si ritiene più efficacemente espletabili al di fuori dell'uniformità burocratica e della logica autoritativa degli apparati amministrativi dello Stato e, ad un tempo, al riparo da una loro «cattura» ad opera degli interessi in gioco o da interessate manipolazioni partigiane. Nel primo caso, invece, abbiamo posizioni soggettive, pretese e aspettative che sono certo private ma la cui operatività assume sempre un intrinseco rilievo collettivo. Di qui la garanzia - ancorata a precisi valori costituzionali - che il legislatore ad esse conferisce in questa loro duplice e simultanea valenza (privata e collettiva insieme) e che le rende non suscettibili di ponderazione autoritativa e di subordinazione preventiva a specifici indirizzi politici, secondo un modulo che commisuri libertà private a interessi pubblici, poiché la loro rilevanza pubblica è già insita alla loro stessa genesi privata ${ }^{46}$. Pertanto, è un'autorità indipendente $\mathrm{e}$

46 «(...) io tutelo degli interessi privati contro altri privati. Quando questo interesse privato però investe la generalità dei privati e non un singolo privato e quando il danno che da quel privato può essere commesso è tale da poter investire non soltanto il diretto danneggiato, ma l'intero mercato (io intervengo): quindi io faccio una cosa diversa dalla pubblica amministrazione, diversa da quella della discrezionalità amministrativa, tant'è vero che quel danno lo accerto: se quel danno c'è stato io lo accerto esattamente col modulo paragiudiziale con cui lavora un giudice. Io verifico le caratteristiche di un fatto e gli applico la norma, proprio nulla di più banalmente, sillogisticamente giudiziario di questo, se non il fatto che il mio sillogismo è nutrito di analisi economiche, ma questo attiene alle caratteristiche della disciplina, lo stesso fa il giudice americano» (Amato 1996, 15). Amato, significativamente, così prosegue il suo ragionamento: «l'interesse alla concorrenza sul mercato non è molto diverso dall'interesse al rispetto dei contratti, attengono tutt'e due al buon andamento delle relazioni commerciali»; ora «perché ci deve essere un'autorità per il rispetto della concorrenza e non per il rispetto dei contratti: per i quali si va esclusivamente e normalmente dal giudice?». La risposta è che con la violazione di una clausola contrattuale, l'interesse violato è solo ed esclusivamente quello della controparte del contratto, «non c'è un interesse più generale nel singolo caso in gioco, l'interesse più generale entra in gioco quando statisticamente settan- 
super partes, la cui legittimazione ed efficacia arbitrale risiede nella sua estraneità alla responsabilità politica del governo e alla responsività politica del Parlamento, ad essere coerentemente chiamata a dirimere i conflitti tra le parti in gioco e a tutelare il libero confronto e la competizione, pur senza essere un giudice ordinario che decida controversie tra privati, poiché il conflitto $\mathrm{da}$ dirimere ha comunque un'intrinseca consistenza collettiva.

Se ammettiamo che sia questo il modello effettivamente in corso di sperimentazione in Italia, sono almeno due le implicazioni che vanno al momento rilevate. La prima è che - su un piano molto generale ma decisivo per la funzionalità del sistema politico-amministrativo - un simile modello non attenua la politicità intrinseca a qualunque funzione pubblica di regolazione sociale, ma ne «rialloca» le modalità espressive e le responsabilità decisionali. Un «riallocare» in cui è asserita l'imparzialità dell'azione regolatoria, ma che non determina l'auspicata o temuta «neutralizzazione» di detta politicità. Dato che in modo diretto o mediato, le authorities finiscono con lo svolgere, di fatto e comunque, funzioni di coordinamento di un determinato insieme di attività private o di rapporti interprivati, ma pur sempre di rilevanza collettiva. Così come contribuiscono a determinare l'allocazione delle risorse che vi sono connesse e alla prevenzione o composizione dei conflitti (reali o potenziali) che vi risultano correlati ${ }^{47}$. Una funzionalità che mantiene il proprio insopprimibile ancoraggio a quell'authoritative allocation of values che è la missione fondativa e giustificativa di un sistema politico e che conferisce una sostantiva politicità alle issues regolative che le autorità indipendenti debbono trattare. Per quanto se ne allontani la trattazione dalle determinazioni o dall'influenza dell'autorità formalmente politica di un Parlamento o di un Esecutivo, la sostantiva politicità delle diverse materie del contendere, così come quella delle funzioni regolatorie che le assumono ad oggetto, non ne vengono scalfite - né attenuerebbe o «neutralizzerebbe» tale politicità il loro eventuale affidamento al «potere neutrale» di una qualche magistratura pro-

ta contratti su cento sono violati. (...) per la concorrenza non è così: nel momento in cui un'impresa commette un abuso di posizione dominante, il danno che si è verificato ha riguardato l'intero mercato, cioè il danno all'interesse collettivo in questo caso è diretto, non è indiretto» (ibidem).

47 Per parafrasare la definizione che di regolazione sociale danno Lange e Regini $(1987,13)$. 
priamente detta - (Morisi 1995). Anzi, è sempre stato un rischio virtuale, ma immanente, alla strategia istituzionale delle autborities (e la spia dell'illusorietà di un legislatore che postuli la depoliticizzazione delle materie regolande mediante autorità prive di legittimazione politica competenti a regolarle) (Manetti 1994, 101) che, nelle prassi e nelle immagini della indipendenza delle autorità dalle parti in gioco, detta politicità obiettiva si traduca in una surrettizia politicizzazione anche soggettiva di tali istituzioni, quale che sia la natura (tecnica, burocratica o giurisdizionale) della loro formale legittimazione.

La seconda implicazione è che il formato del policy making che si accompagna all'azione regolatoria delle authorities è ontologicamente altro dai modi e dalle logiche del regolare propri dell'esperienza storica italiana, ma anche dai presupposti che ne hanno caratterizzato la forma sostantiva di governo. Il riferimento non è tanto al passato «proporzionalistico» del regime italiano e al suo presente «pre» o «para»-maggioritario, che ha ovvio e assoluto rilievo e che, ove razionalizzato e consolidato normativamente ed elettoralmente, potrebbe o dovrebbe accompagnarsi (secondo auspici autorevoli della dottrina giuscostituzionalistica) alla sostituzione di un «potere spartito tra partiti» con «un potere distribuito per istituzioni indipendenti» (Cassese 1994, 422). Piuttosto, ci si riferisce alla più generale alterità della regolazione mediante authorities rispetto alla ben più radicata e consolidata tradizione italiana di regolazione legislativa degli interessi: basata su una pervasiva normazione provvedimentale, che ha espresso la storica «necessità» del nostro sistema partitico ${ }^{48}$ di strategie distributive di risorse (economiche, politiche, d'autorità), talvolta congiunturalmente compensatorie di rare (in quanto esplicite e all'uopo formulate) opzioni redistributive, e variamente corredate da specifici sostegni istituzionali ed organizzativi. Un'alterità evidente perché, dal lato della «tradizione» italica, abbiamo il raccordo legislativo tra offerta e domanda «efficiente» ${ }^{49}$ di rappresentanza e di consenso, che sostiene e alimenta continuativamente il processo di legittimazione politica e partitica. Dal lato di una regolazione che adotti i

48 Sulla correlazione tra il formato del sistema partitico sviluppatosi nel processo di consolidamento della democrazia italiana e le connotazioni essenziali delle strategie di policy insite nella produzione legislativa, sia consentito rinviare a Morisi (1996).

49 A sostegno o surrogazione, secondo fasi e circostanze diverse, di un'offerta-domanda di rappresentanza «identificante» (Pizzorno 1983b). 
presupposti e i caratteri dell'azione di specifiche autorità indipendenti, abbiamo, invece, apposite e durevoli agenzie, chiamate a interagire direttamente con gli interessi da regolare e con i relativi conflitti, avulse da un mandato politico-rappresentativo o da una responsabilità politica del governo ${ }^{50}$, e che possono muoversi a distanza (virtualmente) «di sicurezza» dagli indirizzi di quest'ultimo (con i quali, anzi, potrebbero benissimo confliggere $)^{51}$. Cosicché, alle forme, alle prassi e agli attori della rappresentanza e dell'intermediazione legislativa, si giustappone e tendenzialmente - si contrappone una pluralità di durevoli arene regolative separate, ove gli attori di specifici «poteri di fatto» e di specifici «poteri di diritto» si confrontano e scambiano «in presa diretta» - le rispettive risorse (legali, aggregative, economiche, cognitive, comunicative) alla ricerca di un qualche «mutuo aggiustamento» (come direbbe Lindblom) tra le proprie missioni e le proprie opportunità nei sistemi di interessi cui appartengono o che, rispettivamente, debbono controllare.

Nella prospettiva di un modello regolativo quale sopra tratteggiato, ci si potrebbe poi chiedere se non siamo forse alla vigilia, pur incerta e ricca di contraddizioni, del formarsi di quel «diverso Stato» di cui parla Bruno Dente in una recente e acuta riflessione sul futuro dello stato amministrativo italiano (Dente 1995). Una delle sue tesi centrali, infatti, sostiene l'illusorietà di un effettivo contenimento dell'ingerenza politica nell'amministrazione, quale perseguito dalle leggi più celebrate dalla cultura giusamministrativistica dei nostri tempi (la legge «142», la «241» e il decreto «29»). Tali innovazioni normative non riproporrebbero altro che un modello antico e irrealistico, nel quale «politica» e «amministrazione» sarebbero separabili in base alla distinzione tra fini e mezzi del policy making: ai politici, la fissazione degli obiettivi; alla burocrazia, la predisposizione degli strumenti per realizzarli. Ma una simile separazione, invece di tutelare l'amministrazione, ne sancirebbe la sostanziale inazione o inefficacia «per il semplice motivo che $\mathrm{i}$ politici non sono quasi mai in grado di fissare i fini (per ragioni non solo pratiche

50 Separatezza che non pare «compensata» - come ho già incidentalmente rimarcato - dalle diverse attribuzioni dei poteri di investitura e di vaglio delle candidature in capo ad organi dell'Esecutivo e del Parlamento, a fronte dell'ampia genericità del mandato operativo che il legislatore tende ad affidare alle authorities.

51 Sulle implicazioni tecniche di tale ipotesi - tutt'altro che scolastica - vedi Morbidelli (1997). 
ma anche teoriche) e che gli obiettivi non possono essere posti indipendentemente dai mezzi, ma vanno dedotti - a posteriori - dagli strumenti a disposizione» (Dente 1995). La soluzione andrebbe rinvenuta, invece, in una ben più solida distinzione di ruoli, basata sulla separazione concettuale e organizzativa tra funzioni preposte alla definizione e alla progettazione della innovazione delle politiche, e funzioni di gestione della loro continuità operazionale. Le prime, affidate alla capacità ricognitiva, propositiva e definitoria della classe e delle istituzioni politiche. Le seconde, attribuite ad un'amministrazione capace, culturalmente e organizzativamente, di assumersi onori e responsabilità non solo gestionali ma anche comprensive della stessa definizione degli obiettivi operativi specifici (Dente 1995, cap. II). Ebbene, proprio un fitto reticolo di agenzie regolative (dislocate su scala nazionale, regionale e locale) dovrebbe sostenere l'azione di un'amministrazione integralmente ripensata nei suoi fondamenti organizzativi e prescrittivi, penetrata e avvolta da una fitta trama di controlli interni ed esterni, miranti a valutare i risultati, gli effetti e l'ottimizzabilità delle sue cognizioni ed opzioni, in luogo della verifica puntuale della legittimità giuridica dei suoi comportamenti. $\mathrm{E}$ in luogo, potremmo aggiungere, del controllo di conformità agli indirizzi dell'autorità politica (o, come si diceva «secoli» or sono, del controllo della «rete delle assemblee elettive»).

\section{Perché le autorità indipendenti}

Se è indubitabile che l'istituzione delle autorità indipendenti sia comunque la risultante della volontà legislativa dell'autorità politica o, almeno, del suo formale sostegno ad ipotesi di ingegneria istituzionale e amministrativa, resta da capire quale sia la ratio di una simile opzione. Sono almeno due le ipotesi di lavoro che potremmo percorrere. Entrambe hanno a che vedere, su un piano più generale, con quel «nuovo modo di essere dei pubblici poteri» in cui si coniugano «sfiducia nello Stato» ${ }^{52} \mathrm{e}$

52 Le cui matrici vengono individuate dalla cultura giuridica in fattori quali: «a) la progressiva crisi dei poteri esistenti (ad esempio dei partiti politici); b) la crisi dello stesso ordinamento statuale, per effetto della regionalizzazione e della sua crescente dipendenza dalla normativa comunitaria; $c$ ) il mutamento delle dimensioni dello Stato. Lo Stato, avendo per così dire "metabolizzato" gli enti pubblici, ha perduto la sua unitarie- 
pluralità dei «luoghi» di conflitto permanente tra interesse pubblico e diritti dei privati, tra interessi diversi della collettività egualmente tutelati da norme e principî costituzionali, tra attività private ed esigenze di specifica tutela della collettività (Perez 1996a, 116-117). Ed entrambe le ipotesi, su un piano più specifico, attengono alla relazione inversamente proporzionale che intercorre tra normazione legislativa e sua traduzione operativa. Un deficit d'efficacia che mette in discussione le modalità più elementari di esercizio del potere pubblico. Formule come lo Stato arbitro o l'État stratège della Francia o dell'enabling state della Gran Bretagna racchiudono questa complessiva difficoltà, al di là delle profonde differenze degli ordinamenti e delle esperienze cui si riferiscono. La strada delle authorities esprime appunto questo disagio, rispetto al quale si propone come una via d'uscita o di contenimento della crisi della legislazione e della capacità degli attori e delle arene che ne assumono la paternità, di controllare e regolare i rapporti e i conflitti che vorrebbero disciplinare.

La prima delle due ipotesi cui $\mathrm{mi}$ riferisco ha carattere storico-analitico ed è fortemente ancorata - ciò che è il suo limite rispetto alla storia delle authorities più antiche - alla specifica produzione legislativa degli anni novanta. Si tratta cioè di verificare e ponderare, al di là delle suggestioni più epiteliali, il grado effettivo di correlazione tra la crisi della «partitocrazia» italiana e del regime democratico che su di essa si fondava (Calise 1994b; Morlino 1996), e gli indirizzi di innovazione politicoamministrativa che, con l'assunto programmatico della separazione tra le funzioni di un'élite politica e quelle di un'élite burocratica, hanno accompagnato il tentativo di una rilegittimazione della classe politica attraverso il suo affermato ritrarsi dall'intermediazione sociale e amministrativa. È l'ipotesi che intravede nella «soluzione» delle autorità indipendenti una specifica opzione di politics of policy making: la ritirata strategica del ceto politico di partito da arene eccessivamente conflittuali per la

\footnotetext{
tà, ma frammentandosi è divenuto oggi più "ingombrante". Sono, infatti, aumentati i soggetti che costituiscono la pubblica amministrazione e sono cresciuti gli interessi, spesso confliggenti, perseguiti dallo Stato, divenuto oggi un organismo multifunzionale e multiorganizzativo; $d$ ) un diverso sentire dell'ordinamento in materia di confini della propria azione, per effetto della emersione dei cd. "nuovi diritti" (ad esempio all'efficienza della pubblica amministrazione) e della maggior tutela da attribuire a diritti esistenti; $e$ ) la necessità di garantire l'attività pubblica dalla crescente pressione di interessi esterni (politici, burocratici e finanziari)» (Perez 1996a, 116-117).
} 
natura degli interessi in gioco, troppo complesse per la sua capacità di «coordinamento», o troppo onerose per la tutela della sua identità od almeno della sua immagine pubblica. Potremmo dire, almeno allusivamente, che è l'ipotesi dell'astensione della classe politica dalla competizione e dal conflitto degli interessi in «regime» di crisi del sistema politico. Potremmo aggiungere, in modo non meno allusivo, che questa astensione può ben sostanziarsi nella ricerca di una nuova supplenza istituzionale e funzionale (appunto le authorities regolative) che, in luogo di quella giudiziaria e dei problemi di «controllo politico» che vi si accompagnano, potrebbe esprimere l'aspettativa dell'attore politico, non importa se illusoria o meno, di mantenere almeno una qualche virtuale capacità di influenza - per quanto indiretta - sulla dinamica degli interessi in gioco. $\mathrm{E}$ ciò, mediante l'attivazione e la legittimazione di specifiche razionalità e di specifici saperi «arbitrali», la cui presunta tecnicità dovrebbe garantirne la formale oggettività e insindacabilità di merito, senza tuttavia i più rigidi diaframmi, anche ideologici, che il principio della divisione dei poteri interpone tra classe politica e magistratura.

La seconda ipotesi di lavoro è in realtà il volto bottom up della prima. Essa connette il ricorso alla regolazione delle authorities con la lenta ma progressiva erosione del credito politico di cui ha goduto il ceto di partito presso il sistema degli interessi e le loro strutture lungo il processo di consolidamento della democrazia italiana. La crisi della finanza pubblica e le dimensioni dell'indebitamento dello Stato avrebbero sancito, col presente decennio, l'esaurirsi della disponibilità di risorse utili a politiche compensative di natura distributiva, con un connesso affievolimento della delega politica delle esigenze di espressione $\mathrm{e}$ intermediazione degli interessi. $\dot{E}$ in una parola - anche qui appena allusiva - l'ipotesi dell'erosione del gatekeeping partitico ad opera di questi ultimi che, negli interlocutori politici, troverebbero ormai dei mediatori sempre meno solvibili. $\mathrm{Ma}$, anche per tale profilo così come per il precedente, è chiaro che occorrerebbe disporre di un'accurata periodizzazione.

Detto questo, va ripetuto che resteremmo comunque delusi se, alla base dell'istituzione delle autorità indipendenti italiane, cercassimo un chiaro ed univoco progetto strategico. Col senno del poi, potremmo forse rinvenire, per parafrasare un'altra celebre formula di Charles Lindblom, una qualche superiore intelligenza della democrazia che abbia guidato, pur tra consapevo- 
lezze e volontà politiche diverse nelle differenti circostanze, la mano del legislatore verso un disegno di innovazione, pur incrementale, ma netta e durevole. Quando però l'analisi si sofferma sulle singole vicende istitutive, ecco che ci si presenta una pluralità di situazioni di fatto che ne hanno favorito la promozione, e una gamma di soluzioni normative e organizzative che hanno via via caratterizzato l'attivazione delle singole autorità ${ }^{53}$.

Possiamo, ad esempio, privilegiare la prospettiva più «contingente» del presente decennio - anche se è una contingenza molto «strutturale», connessa com'è a un mutamento in fieri del regime democratico. Se, come oggi comunemente si ritiene, pensiamo che l'istituzione delle authorities sia l'indicatore importante di una modificazione rilevante della forma materiale di governo destinata a ridimensionare, sul piano delle politiche, il peso che il nostro party government vi ha storicamente esercitato, dobbiamo constatare che si tratta di un processo già marcatamente avviato allorché le forme e le logiche del governo di partito erano ancora robustamente in vigore ${ }^{54}$. La stratificazione incrementale della normazione specifica è stata molto spesso il portato di quelle peculiari interazioni di policy making che il supposto «legislatore» intendeva indirizzare e da cui è risultato, invece, «catturato» nelle sue determinazioni normative (La Spina 1996, 295). Tuttavia, oggi, superata la metà degli anni novanta e dato il proliferare di questo genere di istituzioni e il diffondersi della loro progettazione, le spiegazioni più congiunturali hanno di certo molti e buoni argomenti a proprio favore, tanto che sono ormai assunte, anche esplicitamente, tra $i$ pre-

53 Anzi, andando un po' in giro per il mondo, si potrebbe scrivere una sorta di storia infinita di vicende peculiari. Ad esempio, Majone e La Spina $(1991,39)$ ricordano la vicenda del Kartellgesetz «adottata nella Germania federale del 1957, che vietava le concentrazioni fra imprese e introduceva una agenzia regolativa specializzata, il Bundeskartellamt, con poteri ispettivi e amministrativi. Tale "anomalia" va ricondotta alla forte influenza esercitata dagli Stati Uniti, che durante l'occupazione bandirono i cartelli fra industrie tedesche, ritenute responsabili di aver contribuito al consolidamento economico del nazismo». L'influenza degli Stati Uniti in Germania avrebbe continuato pressantemente ad esercitarsi in funzione di una normativa anti-trust sotto la minaccia di una specifica normativa promulgata d'autorità ad opera dell'occupante, fino all'approvazione di un apposito progetto di legge, largamente rivisto da esperti Usa, anche se poi emendato in molte disposizioni decisive per la contropressione degli industriali tedeschi, attraverso l'introduzione di «numerose eccezioni, che in buona misura lo vanificavano» (ibidem).

54 Emblematico è il caso della genesi e dell'evoluzione legislativa della Consob, per le quali rinvio alla ricostruzione che ne ha dato La Spina $(1996,281$ ss.). 
amboli della legislazione promossa in materia in questi anni ${ }^{55}$. Sembra anzi diffusamente accolta l'opinione di quella dottrina costituzionalistica per la quale la motivazione del ricorso alle authorities sarebbe «essenzialmente quella di limitare il potere dell'Esecutivo (...) in quanto organo espressivo di determinate maggioranze politiche. Una motivazione, dunque, che si muove soprattutto sul terreno dei rapporti tra governo e Parlamento, ovvero tra maggioranza e opposizione, ma che assume caratteri specifici», poiché «il tentativo non è tanto quello di limitare i poteri del governo, quanto piuttosto quello di limitare il potere dei partiti e di ricondurre in sedi istituzionali deputate decisioni che una prassi deviata ha progressivamente finito per riservare ai partiti stessi» (Caretti 1995, 194). Ad un tempo, è altrettanto comune la valutazione per la quale le authorities esprimerebbero anche l'intento, collegato al precedente, di ridefinire e reimpostare su nuove basi le relazioni tra competenze «propriamente» politiche e competenze «propriamente» amministrative, al fine di modificare un rapporto tra le due sfere «ancora troppo legato alla tradizione ottocentesca e ancorato all'idea dell'esistenza di un "continuum" tra decisione politica e azione amministrativa, che vede risolta ogni mediazione nella prima e lascia alla seconda un ruolo meramente applicativo-esecutivo. Un'impostazione sempre più difficile da conciliare non solo con lo straordinario arricchimento quantitativo dei compiti dell'amministrazione, ma anche con la profonda trasformazione qualitativa delle sue funzioni, con l'appannarsi del suo carattere prevalentemente se non esclusivamente autoritativo e l'accentuarsi, invece, di una funzione di mediazione (sia pure di secondo livello), spesso di tipo regolamentare o arbitrale, degli interessi di volta in volta in gioco» (Caretti 1995, 195; Cerulli Irelli 1995, 92). Entrambi i moventi starebbero poi in stretta correlazione funzionale a quel processo, lento e vischioso ma pervasivo - almeno come ideologia economico-istituzionale «post-welfare» di riconversione della natura e delle modalità giuridiche dell'in-

55 Cfr., ad esempio, la proposta di legge C.n. 2258 (Strada e altri) dell'XI legislatura, recante «Norme per la regolazione delle tariffe e il controllo della qualità dei servizi di interesse pubblico a tutela degli interessi e dei diritti degli utenti»; il disegno di legge (governo Amato) C.n. 2579, della stessa legislatura, recante «Delega per l'istituzione delle agenzie per i servizi pubblici»; il disegno di legge parlamentare presentato al Senato il $1^{\circ}$ giugno 1994 (Cavazzuti e altri) recante «Norme per la concorrenza e la regolazione dei servizi di pubblica utilità. Istituzione dell'Agenzia di regolazione dei servizi di pubblica utilità». 
tervento pubblico nei mercati di produzione ed erogazione di beni e servizi materiali e immateriali. Un processo in cui privatizzazioni, delegificazione e deregolazione sono le parole d'ordine di quel macro «passo indietro» che autorità dello Stato, della politica e dell'amministrazione dovrebbero compiere - in una direzione, si può ribadire per inciso, di orientamento inverso rispetto ai moventi storici di fondo dell'esperienza «fondativa» statunitense - per liberare dall'inconsistenza genetica dell'«interesse generale» e dall'inesigibilità della responsabilità politica e amministrativa, l'efficienza, la trasparenza e la concorrenzialità dell'agire imprenditoriale, insieme ai diritti al controllo, all'accesso e alla scelta dei cittadini come acquirenti e fruitori informati e contestativi di quegli stessi beni e di quei medesimi servizi (Napolitano 1996).

Eppure, simili spiegazioni - ormai tanto consolidate da risultare quasi di senso comune - riflettono una inevitabile razionalizzazione modellistica ex post del fenomeno, più che una sua sistematica verifica diacronica. Invece, sarebbe almeno utile una angolazione meno contingente $o$ non solo ancorata alla congiuntura storica più immediata.

\section{Una prospettiva di medio periodo}

A questo fine, va ribadito che i processi di policy cui ineriscono le autorità indipendenti sono quelli che la vicenda repubblicana del nostro sistema politico, per almeno quattro decenni, aveva correlato, sia nell'alveo incrementale della «decisione» che in quello della «non decisione» - e pur tra peculiari e corposissime eccezioni (vedi per tutte la centralità macroeconomica della banca centrale) e con efficacia sempre problematica -, alla funzionalità del party government nello sviluppo del processo di consolidamento della democrazia italiana. Cioè, a quell'utilizzazione delle istituzioni rappresentative e di governo e delle loro risorse espressive, amministrative e allocative con cui, a partire dalla Democrazia Cristiana e in sinergia e/o concorso competitivo con essa, la classe politica ha potuto costruire o consolidare la sua rete di alleanze sociali, riconoscendo agli interessi sostenuti il libero perseguimento dei propri fini - nei modi e secondo le convenienze da essi preferiti - annodando attorno a se stessa «l'autogestione del capitalismo, il protezionismo liberale, la società frammentata in miriadi di gruppi di in- 
teresse che negoziano benefici privati» (Amato 1977, 4). È un risultato notoriamente ottenuto mediante il combinarsi di diverse strategie di party government con le modalità che ha storicamente assunto la competizione politica nel nostro paese e con i diversi piani in cui essa si è svolta.

Sappiamo bene, infatti, come sul piano delle «metapolitiche» (Cotta 1996, 29) - quelle cioè che attengono alle opzioni fondamentali circa il regime politico nelle sue correlazioni culturali e funzionali con la società politica e la società civile, e con la collocazione internazionale del paese - la competizione tra i partiti italiani abbia assunto connotazioni fortemente ideologiche e simboliche, con fondamentali, e fondativi, discrimini tra «vinti» e «vincitori» nel medio e nel lungo termine ${ }^{56}$. E sappiamo anche come questo livello «alto» delle politiche pubbliche si sia strumentalmente connesso al piano delle «micropolitiche» (Cotta 1996, 30), cioè alle scelte che intervengono sulle situazioni, le opportunità e i rapporti sociali esistenti mediante incrementali modificazioni al margine, orientate a gestire e/o tutelare specifici e particolaristici interessi di piccoli gruppi categoriali e/o territoriali (vedi, in primis, tutto il capitolo delle «leggine»). Così, sulla base della legislazione elettorale, da un lato, e in funzione, dall'altro, di esigenze di compensazione delle fratture insite nella conflittualità del primo livello, i disegni di policy sono stati fortemente improntati ad attività di patronage distributivo (Blondel e Cotta 1996) che, in quanto tali, non pregiudicavano le immagini e le posizioni dei rispettivi attori in materia di «scelte di civiltà», ed erano dunque agevolmente adottabili col concorso - diretto o indiretto - ma ampio dell'intero spettro partitico ${ }^{57}$. Nel «mezzo» - tra questi due poli del policy making - sarebbe rimasto a lungo sterile (pur con importanti eccezioni) o compresso entro specifiche urgenze congiunturali, il campo di quella corposa e decisiva sfera di issues strutturali che possiamo

56 Discrimini che Cotta $(1996,31)$ ritiene abbiano «a lungo egemonizzato la politica visibile tenendo banco, anche se con caratteri via via meno intensi, fino agli anni settanta (e, a dire il vero, producendo ancora qualche soprassalto negli anni ottanta)».

57 Anche perché «gli attori dei due livelli estremi di competizione tendevano ad essere almeno parzialmente diversi: $i$ partiti identificati dal loro centro nazionale nel primo caso», quello delle «metapolitiche», «molto più spesso invece attori subpartitici locali o settoriali o addirittura individuali» nel caso delle «micropolitiche», anche se «naturalmente il partito non spariva completamente anche su questo piano, ma tendeva ad assumere il significato di regolatore, o di involucro, che delimita i margini di azione degli attori subpartitici, piuttosto che di centro propulsore» (Cotta 1996, 32). 
designare come «mesopolitiche» (Cotta 1996, 29). Vale a dire le opzioni che non toccano le policies di livello superiore, ma che innovano significativamente, e per il lungo termine, ambiti decisivi della vita economica, sociale, istituzionale, internazionale: il regime e l'amministrazione del prelievo fiscale; il quantum di pubblico e privato nella finanza e nell'industria, così come nei servizi di utilità collettiva; l'organizzazione del sistema sanitario, scolastico e previdenziale; il sistema elettorale; il finanziamento dell'economia e la gestione del risparmio delle famiglie; il governo urbanistico del territorio e del regime dei suoli; e via dicendo. Politiche che presuppongono divergenti definizioni dei problemi di lungo andare, e non meno divergenti opzioni risolutorie, in funzione degli interessi da privilegiare o penalizzare o comporre o ponderare.

Si può certo concordare con quanti ritengono che proprio le politiche di questo genere abbiano pagato il prezzo di questo assetto dei livelli di competizione interpartitica, scontando un duplice stato di «sofferenza» decisionale. Da una parte, infatti, si è registrato il frequente gravitare di queste politiche nell'orbita delle metapolitiche, con ricorrenti eccessi di ideologizzazione e non meno ricorrenti propensioni della classe politica a rimuovere o a procrastinare, nelle rispettive agende, simili tematiche, o a frammentarne e diluirne la portata decisionale entro le prassi delle micropolitiche «per non rischiare di mettere in pericolo le posizioni raggiunte nella competizione sul primo livello» (Cotta 1996, 32) ${ }^{58}$. Dall'altra parte, il parallelo radicarsi della frequentazione consociativa delle micropolitiche ad opera dei partiti, ha prodotto vincoli sostantivi, a lungo insormontabili, alla possibilità di trattare i relativi ambiti di intervento decisionale nei termini di una qualche ridefinizione o razionalizzazione settoriale: per la «obiettiva» difficoltà di invertire o correggere aspettative, tutele e rendite particolaristiche, reiteratamente legittimate e pervasivamente alimentate mediante le politiche di taglio micro. Di qui un ridursi dell'attività dei partiti al rinnovo ritualistico degli impegni elettorali «per poi montarvi la guardia in sede di policy making» al fine di cogliervi le virtuali opportu-

58 Ove gli schieramenti elettorali e le alleanze di governo «dettati principalmente da scelte relative al livello delle metapolitiche, richiedevano necessariamente che gli aderenti acconsentissero a mettere la sordina agli orientamenti programmatici divergenti là dove questi potevano essere più significativi, ossia sul piano delle politiche intermedie» (Cotta 1996, 32). 
nità di patronage (Cotta 1996, 33). E di qui, soprattutto, gli amplissimi spazi lasciati alla vicaria funzionalità di istituzioni, arene interattive e circuiti negoziali dotati di risorse di legittimità e di autorità autonome, e caratterizzati da imprenditori politici alternativi a quelli di formale ascendenza democratica. D'altra parte, non era precisamente questo il terreno di policy su cui i partiti - a suo tempo - «avevano definito la propria identità né su questo piano avevano ottenuto un mandato politico dagli elettori. Non avevano dunque né forti risorse di empowerment da spendere né vincoli stringenti a cui attenersi in questo campo. Questa situazione inevitabilmente si rifletteva sulle stesse capacità operative dei partiti. In assenza di una vera necessità esse tendevano a essere del tutto inadeguate alle necessità di elaborazione e proposta di politiche di medio livello» (Cotta 1996, 34).

E chiaro, tuttavia, che un simile assunto può essere condiviso solo con qualche cautela. Non c'è dubbio, è appena il caso di rammentarlo, che il sistema politico italiano abbia conosciuto le proprie cicliche «stagioni di riforme» - tristi o felici, non importa rilevarlo in questa sede. Per le quali (pensiamo, ad esempio, alla fase della seconda metà degli anni settanta) non sono mancati processi decisionali intensamente contrassegnati sia da fortissime prassi di cooperazione interpartitica (secondo una logica che dava compiuta razionalizzazione alla strategia interattiva delle leggine) sia, soprattutto, da intense forme di controllo e di regìa partitocratica (Giuliani 1995, 229). Eppure, al di là dei distinguo storici che quell'assunto può ricevere, esso può comunque ricordarci, una volta di più, che lo spazio occupato dal subsistema partitico (nei suoi formati uni o pluridimensionali, e nei livelli in cui si articola la competizione al suo interno) non si identifica affatto con gli spazi del policy making. E che non v'è nulla di paradossale che anche un regime di party government come quello italiano, così attento e vigile sulle proprie capacità di gatekeeping e sulle proprie virtualità di penetrazione e articolazione degli interessi, abbia manifestato molteplici debolezze e strategiche riluttanze a contrassegnare, col proprio imprinting, aree anche cruciali del policy making ${ }^{59}$. «Debolezze», perché la

59 Anzi, secondo colui che è forse il maggiore esponente della policy analysis italiana «i partiti politici, in quanto soggetti portatori di una visione generale del mondo, o quantomeno di programmi precisi sui quali chiedono ed ottengono il consenso elettorale impegnandosi a tradurre le une o gli altri in politiche pubbliche, non appaiono nel 
presenza e, talora, l'ubiquità istituzionale e sociale di partiti, pur fondativi del sistema politico, non debbono per forza coincidere con una loro rilevanza e una loro continuativa incisività decisionale (Giuliani 1995, 237). «Riluttanze», perché, nella complessità delle società contemporanee, le politiche pubbliche vedono crescere costantemente $i$ loro intrinseci vincoli sostantivi a detrimento della loro fungibilità partigiana. Per cui diventa difficile, per l'attore di partito, calcolare i costi e i benefici - sul piano della coerenza e della identità rappresentativa ma anche su quello della propria dislocazione negoziale e coalizionale - circa il se, il come e il per chi definire e trattare le relative issues.

Così, ad esempio, abbiamo conosciuto politiche industriali «costituite» dal soffocante abbraccio dei partiti al sistema delle imprese e dei servizi pubblici, e dalla loro capillare penetrazione nelle pieghe anche più riposte del management e delle opzioni più minute di politica aziendale ${ }^{60} . \mathrm{Ma}$, ad un tempo, abbiamo anche osservato il costante incremento degli spazi lasciati alla banca centrale nel più penetrante e decisivo governo della politica economica del paese e dei suoi cicli, o a talune burocrazie «tecniche» in settori come la difesa o la gestione della politica estera, oppure alla magistratura nel controllo delle aree e delle forme di conflitto civile o economico più immediatamente connesse alla trasformazione della società e delle sue culture. Tuttavia, sono stati proprio questi spazi autonomi dal controllo partitico - ed è il punto da rimarcare - che si sono rivelati efficaci per la stessa longeva robustezza della classe politica e delle sue formazioni organizzative nella Prima repubblica (robustezza che non è certo venuta meno solo per le debolezze o le difficoltà nell'adottare politiche di «qualità»). E ciò, proprio in virtù della tendenziale autolimitazione - se non autoesclusione - con cui i

nostro paese una realtà». Nei saggi - che formano la prima rassegna sistematica, settore per settore, del policy making italiano, curata da Bruno Dente - «vi è una costante denuncia della latitanza dei partiti, del loro non essere presenti, in quanto soggetti coerenti e organizzati, nelle arene nelle quali si decidono le policies» (Dente 1990, 28).

60 «La politicizzazione del sistema delle imprese pubbliche nella spartizione delle cariche di vertice, ma anche intermedie e inferiori, sino agli infimi livelli, la clientelizzazione delle scelte di assunzioni di settori di attività, come di localizzazione di stabilimenti e via dicendo, con le conseguenti diminuzioni di efficienza e di competitività, e con le successive perdite divenute insostenibili per il bilancio pubblico stremato da sprechi, dispendi, distruzioni di patrimoni, hanno fatto naufragare la concezione dello Stato imprenditore come Stato che all'amministrazione tradizionale e agli equilibri tradizionali fra Stato e non Stato, sostituisca una correzione del mercato affidata alle articolazioni statali al di fuori dell'apparecchio regolatore, cioè alle imprese» (Predieri 1997a, 47). 
partiti hanno interpretato il loro ruolo rispetto alle politiche che in quegli spazi si agitavano. Ma spazi, va ancora ribadito, nient'affatto «neutrali»: né per la rilevanza e la conflittualità delle tematiche che vi si sono affrontate, né per le soluzioni e i disegni di policy con cui quegli attori vicarî le hanno definite, articolate e trattate. La non politicità democratica dell'investitura e della legittimazione di un policy maker e delle interazioni che esso può promuovere o in cui è chiamato ad operare, in luogo o in latenza dell'attore formalmente politico e propriamente di partito, non si vede perché dovrebbe effettivamente tradursi nella neutralizzazione della discrezionalità politica delle funzioni espletate da un attore alternativo, o nella imparzialità, rispetto agli interessi in gioco, del suo agire. La sua azione, in quanto vi sia, non può che esprimere il suo non astenersi dallo scegliere $\mathrm{e}$ dal decidere, ed è perciò stesso non neutrale e sottende sempre un indirizzo politico, una determinata visione strategica o una specifica ponderazione degli interessi coinvolti ${ }^{61}$.

Dunque, se è molto notevole la proliferazione incrementale delle authorities nell'attuale fase della nostra storia politico-istituzionale e se è netta la cesura che le dimensioni della loro diffusione pongono tra il presente decennio e l'esperienza repubblicana antecedente, la loro vicenda non va letta soltanto nel tempo breve della crisi della Prima repubblica e nella indeterminata transizione degli anni novanta, ma va anche collocata nel tempo lungo del processo di consolidamento della democrazia italiana e dei suoi lasciti. Le odierne autorità indipendenti, infatti, hanno più di una parentela con la lunga vicenda di questi autonomici spazi politico-amministrativi, ove sono stati controllati e indirizzati molti e rilevanti processi di policy e dove la (pur non neutrale) funzionalità delle arene e degli attori che li hanno occupati ha conferito stabilità e persistenza alle capacità «gestionali» del sistema, nonostante le turbolenze delle sue congiunture partitiche e governative, e ne ha garantito una durevole operosità decisionale e amministrativa. Sono, anzi, quegli spazi più legati alle politiche di medio o secondo livello (se adottiamo la tipologia poco sopra richiamata) che paiono allargarsi nella transizione critica del sistema politico della Prima Repubblica e trovare una specifica istituzionalizzazione nelle autorità indipendenti.

61 Sulla nozione di neutralità e sulle relative problematiche concettuali in prospettiva politico-istituzionale, cfr. per tutti Predieri (1997b). 
Le quali, pertanto, sviluppano logiche e funzionalità integrative ereditate da quel sistema politico, anche se su di esse si innestano oggi nuovi motivi di specifica legittimazione. Uno di questi è, senz'altro, il ripensamento diffuso e radicale, che del processo di transizione è parte costitutiva, circa i limiti e il realismo di aspettative credibili entro cui il potere pubblico e il governo politico possono aspirare ad una qualche efficacia orientativa nei processi di trasformazione delle società nazionali e in quelli di integrazione sovranazionale dei relativi sistemi economici. Un motivo ulteriore, e in simbiosi col primo, è quella dottrina della stabilità ${ }^{62}$ che fa ormai da sostrato culturale comune a gran parte della classe politica e della pubblica opinione europea (Manzella 1997) e che conforma o sussume in sé le parole e le idee dello sviluppo economico e delle sue «compatibilità», della innovazione sociale e delle sue cautele compensative, dell'assetto istituzionale e dei suoi equilibri pluralistici ${ }^{63}$.

$\grave{E}$ in questa duplice chiave e proprio in quegli spazi non funzionali al primato rappresentativo e mediatorio di attori politico-partitici, che possono mettere o rinsaldare le proprie radici istituzioni come le authorities, che consentano al sistema politico-amministrativo una continuità di interazione e di indirizzo verso gli insiemi della società civile, e contribuiscano, per questa via, ad una complessiva missione di stabilizzazione del sistema politico stesso, pur nel corso di una critica transizione ad un suo nuovo formato istituzionale e nel corso di una connessa e

62 Per la cui genesi politico-economica e la sua articolazione prescrittiva, cfr. Vassallo (1997).

63 «La stabilità diventa un valore che connota un'epoca, che non è né solo italiano né solo europeo (...), che accomuna i paesi industriali e postindustriali, i paesi (relativamente) soddisfatti, e li contrappone in modo che potrà divenire drammatico a quelli che vogliono uno sviluppo, anche con grossi costi politici e sociali. La stabilità, dunque, è valore e bene che il sistema cerca di preservare in ogni modo, non sempre con buoni risultati. La nostra esperienza ha fatto conoscere una stabilità ricercata con un consociativismo mediocre, cioè una istituzionalizzazione dei partiti come centro di potere statico, che rinunzia ai ruoli dinamici di governo da un lato e di opposizione dall'altro, con incontri di compromesso, quindi con un tentativo di sostanziale rafforzamento con neutralizzazione politica finita male perché il metodo ha incrementato corruzione e inefficienza. Anche in questo caso, segnali di una società che strutturalmente tende all'instabilità non mancano, ma ad oggi vengono percepiti non come dati di un mutamento, pur se esso può apparire forse più consono alla nostra epoca in un mondo che sta in un «universo fisico (che) non ha più come simbolo il moto regolare e periodico dei pianeti, moto che è alla base stessa della meccanica classica. Ė invece un universo di instabilità $e$ fluttuazioni» (secondo la formula di Nicolis e Prigogine 1991, XI - orig. 1987 -) che rappresentano motivi di preoccupazione, per non dire incubi, contro cui vengono erette dighe e baluardi, fra cui, anche, le difese di cui parliamo» (Predieri 1997a, 138). 
complessiva ridefinizione e redistribuzione dei poteri di decisione, delle capacità di influenza, delle opportunità e delle tutele sociali.

Una stabilizzazione, va aggiunto, che, se sul piano congiunturale è efficace in quanto delimita il mandato espressivo e negoziale degli attori politici, preservandone funzioni e identità da aree di policy di più difficile e onerosa complessità di scelta e mediazione, su un piano più strutturale è efficace in quanto conferisce stabilità anche al sistema nel suo insieme e all'insieme di relazioni che ne compongono l'ambiente sociale ed economico. Ed è appunto in questo doppio grado di efficacia che può consistere il rendimento di istituzioni «non maggioritarie», disancorate da concezioni monocentriche dei pubblici poteri e da visioni monolitiche della responsabilità politica (Majone 1994). Ispirate, piuttosto, ad un costituzionalismo pluralistico di matrice «lockiana», esse sono in grado di ingerirsi - per aree cruciali del policy making nazionale e sovranazionale - col proprio formato organizzativo, le proprie funzioni ed i propri procedimenti, nella complessità dei processi sociali ed economici e nella complessità del confronto che ne deriva tra governanti e governati. D'altra parte, è proprio questa complessità che le forme della aggregazione e dell'intermediazione politica, a base democratico-elettiva, sempre meno riescono a interpretare e comporre $^{64}$. Essa risulta meglio trattabile, e integrabile in una funzionalità complessiva e articolata del sistema politico-amministrativo, mediante istituzioni che rendano concreta e immediata la «responsabilità» del potere pubblico, non demandandola alla generica indeterminatezza della (presunta) sanzionabilità elettorale, ma garantendola con la partecipazione negoziale o in

64 «Lo spazio autonomo che ogni comunità particolare, fino all'individuo stesso, ormai occupa, e la miriade infinita di decisioni divergenti che ne scaturisce, ai fini della governabilità dell'insieme rende del tutto inservibili i meccanismi tradizionali di decisione e di comando. Muovendo le pedine dell'apparato di governo, la capacità di governare effettivamente non supererà il 20 per cento. In altri termini: se i governati sono essi stessi governanti, una quota rilevantissima di decisioni finisce nella paralisi, o può mettere capo a sussulti improvvisi e scoppi violenti, brevi o duraturi che siano, comunque incontrollabili. (...) La governabilità diventa, per necessità tecnica, anche autogoverno. (...) il mandato riconosciuto alla politica non è più onnicomprensivo, diventa sempre più un mandato limitato. (...) Non è un caso, infatti, che in tutto l'Occidente cresca la richiesta di autorità indipendenti. La richiesta, cioè, che aree un tempo affidate alla decisione politica, per la delicatezza dei diritti e delle libertà fondamentali in esse in gioco, vengano affidate all'imparzialità di un arbitro sottratto alla politica. Ma crescono anche gli ambiti in cui l'individuo rivendica che la decisione non possa che essere etica, dunque affidata alla sua autonoma responsabilità» (Amato 1997b, 236-237). 
contraddittorio dei diretti destinatari dell'attività regolativa, e con la trasparenza del suo decision making e delle sue interazioni con i portatori di interessi e di diritti specifici.

Non a caso, nella dottrina giuridica che studia le autorità indipendenti, è ricorrente il tema delle assonanze tra le modalità e le logiche decisionali del loro agire e quelle proprie della funzione giurisdizionale. Assonanze che vengono sovente interpretate come una risposta istituzionale e organizzativa «al netto venir meno della fiducia nel carattere politico rappresentativo delle autorità amministrative quale garanzia di una amministrazione corrispondente alle aspettative ed alle esigenze della società» (Falcon 1993, 95). E che, a impostazioni meramente «burocratiche» o «tecnocratiche» ${ }^{65}$, sembra preferire «il modello di una amministrazione in senso amplissimo «giusdicente» (uno jus dire comprensivo sia del «regolare» che del «decidere») in funzione obiettiva - anche se non in posizione terza o neutrale, ma nel perseguimento di un interesse assegnato - da affidarsi a persone in grado di figurare come «autorità» per le loro stesse caratteristiche intellettuali, sociali, morali, prima ancora che per le funzioni pubbliche loro attribuite» (Falcon 1993, 96) ${ }^{66}$. Un modello di «amministrazione giusdicente», si può aggiungere, la cui autonomia e indipendenza trova proprio, e significativamente, nell'indipendenza e nell'autonomia del potere giudiziario il solo eventuale controllo di coerenza tra le modalità empiriche dell'azione delle autborities e la loro alterità sia dal potere politico che dagli interessi che debbono regolare. Per cui, proprio perché «le autorità indipendenti, escluse dal circuito elettorale direttamente $o$ indirettamente, non democratiche quindi, non rispondono se non ad un sistema non responsabile democrati-

65 Che tuttavia non vengono escluse in settori anche cruciali del sistema amministrativo, come il servizio sanitario nazionale e a cui si ispira lo stesso processo di riforma del pubblico impiego.

66 Sulle parentele di un simile modello con «organi di origine ben più antica, che già in passato ne incarnavano alcuni tratti» - le cosiddette amministrazioni contenziose - «che vennero proprio per questo discussi e distinti nell'ambito degli apparati amministrativi e che alcuni, oggi, considerano $i$ loro antenati [delle autorità indipendenti], soprattutto per la loro (irrealizzata) potenzialità eversiva rispetto alla uniforme modellistica» degli apparati amministrativi, e sulla distinzione concettuale, che ne individuava la specificità funzionale, «fra attività provvedimentali, volte a soddisfare interessi concreti in situazioni variabili e attività «dichiarative», volte ad applicare il diritto positivo a fattispecie invariabili e terminate e produttive perciò, a differenza delle prime, di atti suscettibili di assumere lo stesso valore della cosa giudicata», cfr. Amato $(1996,18)(\mathrm{da}$ cui ho tratto i brani testé citati), e Cassese $(1996,217)$. 
camente, né nominato democraticamente» (Predieri 1997a, 122123), esse possono comunque agevolare l'innovazione e il mutamento del regime democratico, in quanto contribuiscono al permanere delle sue capacità di regolazione sociale ${ }^{67}$.

\section{Per concludere}

Se ipotizziamo questa specifica funzionalità sistemica delle autorità indipendenti alla stabilità del sistema politico amministrativo e alle sue attitudini ad integrare, nel proprio funzionamento e nelle proprie articolazioni organizzative e decisionali, la complessità dei processi sociali ed economici, non possiamo «classificarne» l'esperienza entro modelli teorici (ancorché autorevolissimi, se non classici) che coniugano l'operatività di (presunti) «poteri neutrali» a fasi e processi di criticità dei regimi democratici $^{68}$. Sia per le specificità istituzionali e funzionali delle autorità indipendenti rispetto alle entità e ai soggetti cui di norma ci si riferisce in quella modellistica, sia - soprattutto perché le istituzioni di cui abbiamo parlato appartengono alla longue durée del sistema, e ne esprimono le endogene capacità di autocorrezione e adeguamento ai mutamenti ambientali, piuttosto che le sue eventuali patologie strutturali. Ed è in questa chiave che è opportuno analizzare empiricamente l'esperienza delle authorities italiane, osservare come e quanto le più recenti tra esse si vadano istituzionalizzando, valutarne di fatto la forza e il rendimento istituzionale, ponderare l'impatto della loro azione nei subsistemi cui sono deputate e sul funzionamento complessivo del sistema politico-amministrativo.

\section{Riferimenti bibliografici}

Amato, G. (1977), Elaborato di sintesi, in Centro Nazionale di Prevenzione e Difesa sociale, Conflitti sociali e mediazione parlamentare in Italia, Roma, ciclostilato.

67 C'è chi, su questa strada, si spinge ad immaginare - in una prospettiva comparatistica americanocentrica - il formarsi di una alleanza tra corti e regolatori, ove le prime consentirebbero l'efficacia degli indirizzi regolativi perseguiti dai secondi a prescindere dall'influenza partigiana del capo dell'Esecutivo, per cui i giudici assurgerebbero al ruolo di tutori dell'indipendenza dei regolatori (Majone 1994).

68 Cfr. per tutti Linz $(1981,141$ ss.). 
- (1996), Le autorità indipendenti, relazione al Convegno organizzato dalla «Rivista trimestrale di diritto e procedura civile» sul tema Le autorità amministrative indipendenti, Bologna 14 dicembre.

- (1997a), Intervento alla Giornata di studi organizzata a Firenze dal Cesifin il 16 febbraio 1996 su Le autorità indipendenti nei sistemi istituzionali ed economici, in AA.VV., Le autorità amministrative indipendenti nei sistemi istituzionali ed economici, Firenze, Passigli.

- (1997b), Intervento, in P. Flores d'Arcais e G. Amato, La sinistra dopo $i$ socialismi, in «MicroMega», 1, pp. 236-237.

Autin, J.L. (1991), Le contrôle des autorités administratives indépendantes par le Conseil d'Etat est-il-pertinent?, in «Revue de droit publique», n. 6, pp. 1533-1563.

Belligni, S. (1990), Lineamenti di scienza politica, Milano, Angeli.

Blondel, J. e M. Cotta (a cura di) (1996), Party and Government. An Inquiry into the Relationship between Government and Supporting Parties in Western Liberal democracies, London, Macmillan.

Bobbio, L. (1986), Uno shock per rifare lo stato, in «L'indice dei libri del mese», XIII, n. 1, p. 6.

Calise, M. (1994a), Da sponda a sponda: la metamorfosi corporativa tra Europa e America, in AA.VV., Storia, Amministrazione, Costituzione, Annale Isap, 2/1994, Bologna, Il Mulino.

- (1994b), Dopo la partitocrazia. L'Italia tra modelli e realtà, Torino, Einaudi.

- (1997), Le Corporations come autorità indipendenti. Alle origini della regolazione economica in America, relazione tenuta alla Giornata di studi organizzata a Firenze dal Cesifin il 16 febbraio 1996 su Le autorità indipendenti nei sistemi istituzionali ed economici, in AA.VV., Le autorità amministrative indipendenti nei sistemi istituzionali ed economici, Firenze, Passigli.

Caretti, P. (1995), Relazione introduttiva, alla parte III del Convegno sulle autorità amministrative indipendenti, dedicata a $I l$ quadro italiano: le prospettive di riforma, promosso da «Problemi dell'informazione».

Cassese, S. (1991), Stato e mercato, dopo privatizzazioni e deregulation, in «Rivista trimestrale di diritto pubblico», n. 2, pp. 378-387.

- (1994), La Commissione Nazionale per le Società e la Borsa - Consob e $i$ poteri indipendenti, Relazione alla cerimonia per il ventennale della legge istitutiva della Commissione nazionale per le società e la borsa, Roma, 7 giugno 1994, pubblicata in «Rivista delle società», pp. 412-425.

- (1995), Il ruolo e la collocazione dei poteri indipendenti nell'ordinamento, relazione tenuta al Convegno internazionale sulla concorrenza «Francesco Saja», svoltosi a Roma il 20 e il 21 novembre 1995.

- (1996), Conclusioni, in S. Cassese e C. Franchini (a cura di), I garan- 
ti delle regole. Le autorità indipendenti nel sistema istituzionale italiano; $i$ loro compiti normativi, amministrativi, giurisdizionali. Un tema centrale per la democrazia del nostro paese, Bologna, Il Mulino. Cerulli Irelli, V. (1995), Relazione introduttiva, alla parte II del Convegno sulle autorità amministrative indipendenti, dedicata a $I l$ quadro italiano: le prospettive di riforma, promosso da «Problemi dell'informazione».

Chevallier, J. (1986), Réflexions sur l'institution des autorités administratives indépendantes, in «La semaine juridique», n. 3254.

Clarich, M. (1993), Per uno studio sui poteri dell'Autorità garante della concorrenza e del mercato, in F. Bassi e F. Merusi (a cura di), Mercati e amministrazioni indipendenti, Milano, Giuffrè.

Colliard, C.A. e G. Timsit (a cura di) (1989), Les autorités administratives indépendantes, Paris, Presses Universitaires de France.

Costi, R. (1995), Libertà di tariffe e concorrenza, in «Diritto ed economia dell'assicurazione», n. 1 .

Cotta, M. (1989), Il contributo dei «policy studies» alla scienza politica contemporanea, in A. Panebianco (a cura di), L'analisi della politica. Tradizioni di ricerca, modelli, teorie, Bologna, Il Mulino.

Cotta, M. (1996), La crisi del governo di partito all'italiana, in Cotta e Isernia (1996), pp. 11-52.

Cotta, M. e P. Isernia (a cura di) (1996), Il gigante dai piedi di argilla. Le ragioni della crisi della prima repubblica: partiti e politiche dagli anni'80 a Mani pulite, Bologna, Il Mulino.

Dahl, R. (1989), Democracy and its Critics, New Haven-London, Yale University Press.

D'Alberti, M. (1995), Autorità indipendenti (dir. amm.), in Enciclopedia Treccani (aggiornamento).

Delle Cave, O. (1994), Analisi delle politiche pubbliche e concorrenza, in «Rivista trimestrale di Scienza dell'amministrazione», LI, n. 3, pp. 5-60.

Dente, B. (1990), Introduzione, in B. Dente (a cura di), Le politiche pubbliche in Italia, Bologna, Il Mulino.

- (1995), In un diverso stato. Come rifare la pubblica amministrazione italiana, Bologna, Il Mulino.

Dicey, A.V. (1962), Law and Public Opinion in England, London, Macmillan.

Diez Picazo, L. (1997), Le autorità indipendenti: profili costituzionali, Lezione tenuta al Seminario di studi e ricerche parlamentari 1996, in corso di stampa per i Quaderni dell'Associazione per gli studi e le ricerche parlamentari, Torino, Giappichelli.

Falcon, G. (1993), Il «primo», il «secondo» ed il «terzo» garante nei settori dell'editoria e della radiodiffusione, in F. Bassi e F. Merusi (a cura di), Mercati e amministrazioni indipendenti, Milano, Giuffrè.

Farneti, P. (1973), Introduzione, in P. Farneti (a cura di), Il sistema politico italiano, Bologna, Il Mulino. 
Ferrari, P. (1997), Agenzismo. La regolazione degli interessi tra passato europeo e modello americano, Milano, Feltrinelli.

Franchini, C. (1988), Le autorità aministrative indipendenti, in «Rivista trimestrale di diritto pubblico», n. 3, pp. 549-583.

Gallino, L. (1976), Sociologia dello Stato, in «Quaderni di Sociologia», n. 4.

- (1987), Della ingovernabilità. La società italiana tra premoderno e neo-industriale, Milano, Comunità.

Genevois, B. (1989), Le Conseil Constitutionnel e la définition des pouvoirs du Conseil Supérieur de l'Audiovisuel, in «Revue Française de Droit Administratif», n. 2.

Giuliani, M. (1995), Le politiche pubbliche fra sistemi di partito e policy networks, in Regonini (1995), pp. 219-248.

Gramsci, A. (1976), Quaderni dal carcere, Torino, Einaudi.

Horn, J.M. (1995), The Political Economy of Public Administration. Institutional Choice in the Public Sector, Cambridge, Cambridge University Press.

Horwitz, R.B. (1989), The Irony of Regulatory Reform: the Deregulation of American Telecommunications, New York, Oxford University Press.

Irti, N. (1992), Società civile. Elementi per un'analisi del diritto privato, Milano, Giuffrè.

Isernia, P. (1995), La cooperazione allo sviluppo, Bologna, Il Mulino.

Krasner, S.D.( 1984), Approaches to the State, in «Comparative Politics», n. 4.

La Spina, A. (1985), La Consob (Commissione nazionale sulle operazioni di borsa), in «Sisifo», 29, p. 11.

- (1996), Partiti e mercato: le politiche di regolazione, in Cotta e Isernia (1996), pp. 273-335.

Lange, P. e M. Regini (1987), Gli interessi e le istituzioni: forme di regolazione sociale e politiche pubbliche, in. P. Lange e M. Regini (a cura di), Stato e regolazione sociale, Bologna, Il Mulino.

Lindblom, C.E. (1965), The Intelligence of Democracy: DecisionMaking through Mutual Adjustment, New York, The Free Press.

- (1968), The Policy-Making Process, Englewood Cliffs, PrenticeHall (I ed.).

Linz, J.J (1981), La caduta dei regimi democratici, Bologna, Il Mulino.

Lowi T.J. (1964), American Business, Public Policy, Case Studies and Political Theory, in «World Politics», 1964, n. 4.

- (1985), The State in Politics: The Relation between Policy and Administration, in R.G. Noll (a cura di), Regulatory Policy and the Social Sciences, Los Angeles, University of California Press.

Machin, H. (1989), L'experiénce britannique, in Colliard e Timsit (1989).

Maisl, H. (1989), Les Autorités Administratives Indépendantes: Protection des libertés or régulation sociale?, in Colliard e Timsit (1989). 
Majone, G. (1992), Cross-National Sources of Regulatory Reform in Europe and in the United States, in «Journal of Public Policy», II, n. 1, pp. 79-106.

- (1994), Independence v. Accountability? Non-Majoritarian Institutions and Democratic Government in Europe, in «EUI Working Papers», SPS, n. 94/3.

Majone, G. e A. La Spina (1990), Deregulation or Re-regulation? Regulatory Reform in Europe and in the United States, London, Pinter.

- (1991), Lo Stato regolatore, in «Rivista trimestrale di Scienza dell'Amministrazione», XXXVIII, n. 3, pp. 3-61.

- (1992a), Deregulation e privatizzazione: differenze e convergenze, in «Stato e Mercato».

- (1992b), Cross-National Sources of Regulatory Reform in Europe and in the United States, in «Journal of Public Policy», II, n. 1, pp. 79-106.

- (1995), La crescita dei poteri regolativi nella Comunità Europea, in «Rivista italiana di scienza politica», XXV, n. 3, pp. 409-439.

Manetti, M. (1994), Poteri neutrali e costituzione, Milano, Giuffrè.

Manzella, A. (1997), Forma di governo e integrazione europea, lezione al Seminario di studi e ricerche parlamentari 1997, in Associazione per gli studi e le ricerche parlamentari, Quaderno n. 8, Torino, Giappichelli.

Massera, A. (1988), «Autonomia» $e$ «indipendenza» nell'amministrazione dello Stato, in Scritti in onore di Massimo Severo Giannini, vol. III, Milano, Giuffrè.

Mitnick, B.M. (1980), The Political Economy of Regulation, New York, Columbia University Press.

Morange, J. (1987), La Commission Nationale des Communications et Libertés e le droit de la communication audiovisuelle, in «Revue Française de Droit Administratif», n. 3.

Morbidelli (1997), Procedimenti amministrativi delle authorities, relazione tenuta alla Giornata di studi organizzata a Firenze dal Cesifin il 16 febbraio 1996 su Le autorità indipendenti nei sistemi istituzionali ed economici, in AA.VV., Le autorità amministrative indipendenti nei sistemi istituzionali ed economici, Firenze, Passigli.

Morisi, M. (1992), Le leggi del consenso, Soveria Mannelli, Rubbettino.

- (1995), Attorno alla legalità come principio, in Associazione per gli studi e le ricerche parlamentari, Quaderno n. 5, Torino, Giappichelli.

- (1996), Il parlamento fatto dai partiti, in AA.VV., Italia 50 anni 1946-1996, Roma, Iscoa, pp. 105-135.

Morlino, L. (1991), Introduzione, in L. Morlino (a cura di), Costruire la democrazia, Bologna, Il Mulino.

- (1996), Crisis of Parties and Change of Party System in Italy, in «Party Politics», II, n. 1, pp. 5-30.

Napolitano, G. (1996), Autorità indipendenti e tutela degli utenti, in «Giornale di diritto amministrativo», n. 1, pp. 14-18. 
Nicolis, G. e I. Prigogine (1991), La complessità. Esplorazioni nei nuovi campi della scienza, Torino, Einaudi (ed. orig. Exploring Complexity. An Introduction, München, Piper, 1987).

Panebianco, A. (1993), Fare a meno della politica, in «il Mulino», XLII, n. 348.

Pasquino, G. (1985), Restituire lo scettro al principe, Bari, Laterza.

Peluffo, P. (1996), La presenza dello Stato nell'economia, in AA.VV., Italia 50 anni - 1946-1996, Roma, Iscoa, pp. 193-199.

Perez, R. (1991), Le Next Steps Agencies e il riordino del sistema amministrativo inglese, in «Rivista trimestrale di diritto pubblico», n. 4.

- (1996a), Autorità indipendenti e tutela dei diritti, in «Rivista trimestrale di diritto pubblico», n. 1.

- (1996b), L'attuazione della riforma amministrativa in Gran Bretagna, in Scritti in onore di Alberto Predieri, vol. II, Milano, Giuffrè.

Pizzorno, A. (1983a), Interests and Parties Pluralism, in S. Berger (a cura di), Organizing Interests in Western Europe: Pluralism, Corporatism, and Transformation of Politics, Cambridge, Cambridge University Press (trad. it. Il sistema pluralistico di rappresentanza, in S. Berger (a cura di), L'organizzazione degli interessi nell'Europa occidentale, Bologna, Il Mulino).

- (1983b), Sulla razionalità della scelta democratica, in «Stato e Mercato», n. 3.

- (1993), Introduzione allo studio della partecipazione politica, in «Quaderni di Sociologia», n. 3-4, poi ripubblicato con il titolo Condizioni della partecipazione politica, in A. Pizzorno, Le radici della politica assoluta e altri saggi, Milano, Feltrinelli.

Poggi, G. (1990), Sociologia dello Stato, in A. Panebianco (a cura di), L'analisi della politica, cit., pp. 293-317.

Portinaro, P.P. (1990), La teoria politica contemporanea e il problema dello Stato, in A. Panebianco (a cura di), L'analisi della politica, cit., pp. 321-335.

- (1995), Sulla legittimità del nuovo, in «Teoria politica», XI, n. 1, pp. 17-44.

Predieri, A. (1963), La produzione legislativa, in A. Predieri, G. Sartori e S. Somogyi, Il parlamento italiano 1948-1963, Napoli, Esi.

- (1975a), Il governo colegislatore, in F. Cazzola, A. Predieri e G. Priulla, Il decreto-legge fra governo e parlamento, Milano, Giuffrè.

- (1975b), Parlamento 1975, in A. Predieri (a cura di), Il Parlamento nel sistema politico italiano, Milano, Comunità.

- (1987), Il legislatore recalcitrante e il rimedio degli effetti diretti delle direttive comunitarie, in Atti del Convegno svoltosi a Firenze su L'adeguamento della disciplina delle società per azioni alle direttive comunitarie nel dPR 30/1986, Firenze, Passigli.

- (1993), Un diritto solidarista contro le diseconomie esterne, in «Studi \& Informazioni. Rivista trimestrale sul governo dell'economia», n. 3 . 
- (1996), Il potere della banca centrale: isola o modello?, Firenze, Passigli.

- (1997a), L'arcipelago delle autorità amministrative indipendenti, Firenze, Passigli.

- (1997b), Carl Schmitt, un nazista senza coraggio, Firenze, La Nuova Italia.

- (1997c), La certa osmosi - Gli incerti paradigmi, Firenze, Passigli.

Regonini, G. (1994), Partiti, reti, giochi, politiche pubbliche, in M. Caciagli, F. Cazzola, L. Morlino e S. Passigli (a cura di), L'Italia fra crisi e transizione, Roma-Bari, Laterza, pp. 163-189.

- (a cura di) (1995), Politiche pubbliche e democrazia, Napoli, Esi.

Romiti, C. (1996), Non sono un pentito di Maastricht, intervista rilasciata a Massimo Giannini in «La Stampa», 5 settembre.

Roppo, E. e R. Zaccaria (a cura di) (1991), Il sistema radiotelevisivo pubblico e privato, Milano, Giuffrè.

Sabourin, P. (1989), Les autorités administratives indépendantes dans l'Etat, in Les autorités administratives indépendantes, Paris, Presses Universitaires de France.

Sacconi, M. (1992), La finanza e la regola. La grande riforma del mercato finanziario in Italia, Milano, Longanesi.

Santoro, E. (1994), Le antinomie della cittadinanza: libertà negativa, diritti sociali e autonomia individuale, in D. Zolo (a cura di), La cittadinanza. Appartenenza, identità, diritti, Roma-Bari, Laterza.

Sartori, G. (1976), Parties and Party Systems, Cambridge, Cambridge University Press.

Schmitt, C. (1972), Le categorie del politico, Bologna, Il Mulino.

Schrameck, O. e X. Delcros (1988), La Commission de Sondages, un garant fragile de la démocratie, in «Act. jur. dr. adm.», n. 6.

Selznick, Ph. (1985), Focusing Organizational Research on Regulation, in R.G. Noll (a cura di), Regulatory Policy and the Social Sciences, Los Angeles, University of California Press.

Teitgen Colly, C. (1989), Les autorités administratives indépendantes: Histoire d'une institution, in Les autorités administratives indépendantes, Paris, Presses Universitaires de France.

Torchia, L. (1996), Gli interessi affidati alla cura delle indipendenti, in $\mathrm{S}$. Cassese e C. Franchini (a cura di), I garanti delle regole. Le autorità indipendenti nel sistema istituzionale italiano; $i$ loro compiti normativi, amministrativi, giurisdizionali. Un tema centrale per la democrazia del nostro paese, Bologna, Il Mulino.

Vassallo, S. (1997), Assetti politico-istituzionali e governo della finanza pubblica. Questioni teoriche e verifiche comparate, dissertazione per il Dottorato di ricerca in Scienza della politica, IX ciclo, Università degli Studi di Firenze, febbraio.

Volpe, G. (1996), Indagine sulle funzioni dell'autorità antitrust: verso un «governo tecnico di settore», in Scritti in onore di Alberto Predieri, Milano, Giuffrè, pp. 1545-1553. 
Wilson, D. (1995), Quangos in the Skeletal State, in «Parliamentary Affairs», vol. 48, n. 2.

Wilson, W. (1885), Congressional Government, Boston, Houghton Mifflin.

Zamagni, S. (1996), Il mercato tra etica e diritto, in «Queste istituzioni», XXIV, n. 105, p. 38.

Zolo, D. (1992), Il principato democratico. Per una teoria realistica della democrazia, Milano, Feltrinelli. 